Mitteilungen aus dem pharmaceutisch - chemischen Institut der Universität Marburg.

\title{
Ueber das Thiosinamin (II).
}

\author{
Von Dr. J. Gadamer.
}

Am Schluls seiner Arbeit verbreitet sich Falke, auf Grund der von ihm angestellten Versuche und untersuchten Präparate, über die Konstitution des durch Einwirkung von Brom auf alkoholische Thiosinaminlösung entstehenden Körpers. Er kommt dabei zu dem Schlufs, dafs dem Thiosinamindibromid keinestalls eine Formel zukommen können, welche der von Andrea s ch für den Monobrompropylenharnstoff acceptierten entspräche, dafs vielmehr auch diese Verbindung sich von der unsymmetrischen Form des Thiosinaming ableiten müsse. Er stellt intolgedessen für das Thiosinaminbromid folgende Formeln aut:<smiles>N=C1NCC(Br)C1Br</smiles>

II.<smiles>CC1SC(=N)NC1Br</smiles>

Hierzu würden sich dann als dritte und vierte Formel die von der zweiten unsymmetrischen Thiosinaminformel (III, l. c.) sich in analoger Weise ableitenden gesellen:

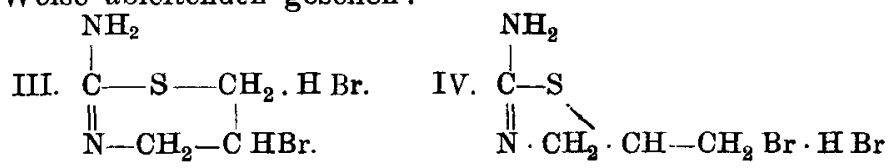

Die zweite Formel (II) würde der von Gabriel für den Propylen $\psi$ thioharnstoff angenommenen Konstitution entsprechen und das Thiosinaminbromid somit als ein Monobromsubstitutionsprodukt desselben aufzufassen sein. Eine definitive Entscheidung, welcher von beiden Formeln der Vorzug zu geben sei, hat Falke nicht getroffen. Zur Entscheidung dieser Frage habe ich das Thiosinamin. 
dibromid selbst und einige Verbindungen desselben, die mir nicht genügend studiert schienen, sowie die entsprechende Jod- und Chlorverbindung einer Untersuchung unterworfen.

Thiosinamindibromid.

Nach der Vorschrift F a lke's tröptelte ich zu einer alkoholischen Thiosinaminlösung mit dem gleichen V'ol. Alkohol verdünntes Brom hinzu. Anfänglich wurde dasselbe sofort unter ziemlich lebhafter Erwärmung addiert, so dals ich, zur Vermeidung einer Zer. setzung, das Reaktionsgemisch gut kühlen mufste. Gegen Schlufs der Reaktion verschwand die Gelbfürbung nur noch sehr langsam, bis endlich, bei einem geringen Ueberscbufs von Brom, eine schwache Gelbfärbung bestehen blieb. Den Alkohol liel's ich nun bei 50 bis $60^{\circ}$ und schlielslich bei gewöhnlicher Temperatnr verdunsten, wobei sich das Thiosinamindibromid in leicht zerreiblichen, undurchsichtigen Krystallen abschied. Der Schmelzpunkt wurde als bei $139^{\circ}$ liegend ermittelt; Falke giebt $138-137^{\circ}$ und $M$ aly $146-147^{\circ}$ an. $\mathrm{F}$ a $1 \mathrm{k} \theta$ erwähnt ferner, dafs das ursprünglich ausgefallene, also nicht umkrystallisierte Krystallmebl, bei sonst gleichem chemischen Verhalten, einen niedrigeren Schmelzpunkt, als das aus verdünntem Alkohol umkrystallisierte Produkt habe. Der Schmelzpunkt desselben liege bei $115^{\circ}$, also $22^{0}$ niedriger, als beim umkrystallisierten Präparat. Falke schliefst daraus, dals bereits durch Umkrystallisieren eine dem ursprünglichen, einfachen Bromadditionsprodukte des Thio. sinamins isomere Verbindung entstanden sei, und glaubt, dals sich zuerst durch einfache Addition von 2 Atomen Brom Dibrompropylthioharnstoff bilde, der durch Umkrystallisieren in das bromwasserstoffsaure Salz des Monobrompropylen- $\psi$-Thioharnstoffs übergebe. Ich kann mich dieser Ansicht $\mathbf{n} \mathrm{i} \mathrm{h}$ t anschlielsen, vermute vielmehr, dafs die Differenz im Schmelzpunkt ibre Erklärung in einer Verunreinigung durch anhaftende Mutterlauge findet. Mein Präparat, dessen Schmelzpunkt bei $1390 \mathrm{lag}$, bestand ebenfalls aus direkt ausgeschiedenen Krystallen; allerdings hatte ich die Lösung bei $50-60^{\circ} \mathrm{kon}$ zentriert, aber auch ein anderes, durch völlig freiwilliges Verdunsten erzieltes Präparat hatte annähernd denselben Schmelzpunkt, nämlich $137^{\circ}$, und bestand aus wasserbellen, schwach gelblich getärbten, bis $3 \mathrm{~cm}$ langen Tafeln, die an beiden Enden zugespitzt waren. Dals auch dieses 
Präparat ein bromwasserstoffsaurer Monobrompropylen- $\psi$-thioharnstoff war, konnte ich, aulser an dem chemischen Verhalten, auch daran erkennen, daís die Krystalle isomorph mit dem durch Umsetzung des Bromids mit Chlorsilber erlaltenen chlorwasserstoffsauren Salze waren.

Die Zusammensetzung des Thiosinaminbromids:

$$
\mathrm{C}_{4} \mathrm{H}_{7} \mathrm{Br} \mathrm{N} \mathrm{N}_{2} \mathrm{~S}, \mathrm{H} \mathrm{Br} \text {, }
$$

sowie die sonstigen Eigenschaften fand ich im Einklang mit den Angaben von $\mathrm{Maly}$ und von Falke (l. c.).

Einwirkung von Goldehlorid a u das Bromid des Thiosinamins.

Versetzt man eine wässrige Lösung des Thiosinaminbromids mit Goldchlorid, so erbält man einen gelbroten Niederschlag, der sich jedoch alsbald in dunkelrote, kleine Krystalle verwandelt. Nach Maly's Angaben beruht die Umwandlung in der Farbe des ausgefallenen Salzes auf einem Austausch von Brom und Chlor, den er in folgender Weise veranschaulicht:

$3 \mathrm{C}_{4} \mathrm{H}_{8} \mathrm{~N}_{2} \mathrm{~S} \mathrm{Br} \mathrm{Br}+\mathrm{An} \mathrm{Cl}_{3}=3 \mathrm{C}_{4} \mathrm{H}_{8} \mathrm{~N}_{2} \mathrm{~S} \mathrm{BrCl}+\mathrm{Au} \mathrm{Br}$.

Falke kommt auf Grund seiner Analysen zu der Ansicht, dafs die von $\mathrm{Maly}$ angegebene Gleichung nicht der Wirklichkeit entspräche, und nimmt an, dafs bei der Einwirkung von Goldchlorid auch das zweite Bromatom gegen Chlor ausgetauscht würde, während $\mathrm{Au} \mathrm{Cl}_{3}$ in $\mathrm{Au} \mathrm{Br}{ }_{3}$ überginge. Diese Annahme hat von vornherein wenig Wahrscheinlichkeit für sich wegen des Verhaltens des zwoiten Bromatoms gegen Chlorsilber, Silbernitrat und starke Salzsäure.

Wie meine Untersuchungen ergeben haben, beruht dieselbe in der That auf einem Irrtum, welcher durch die $z$ a fällig auf die Formel

$$
\mathrm{C}_{4} \mathrm{H}_{8} \mathrm{~N}_{2} \mathrm{~S} \mathrm{Cl}_{2} \mathrm{Au} \mathrm{Br}_{3}
$$

gut passenden Resultate herbeigeführt worden ist.

Eine wässrige Lösung des Bromids versetzte ich mit überschüssigem Goldchlorid; es entstand sofort eine krystallinische Fällung von gelbroter Farbe, die ich durch Absangen sofort von den Mutterlaugen trennte.

Nach dem Pressen zwischen Filtrierpapier, trocknete ich das Salz erst über Schwefelsäure, dann bei 1000 . wobei es jedoch fast nichts an Gewicht verlor. Es war somit krystallwasserfrei. 
1. $0,1702 \mathrm{~g}$ des Salzes hinterlielsen $0,0873 \mathrm{~g}$ Gold

2. $0,2941 \mathrm{~g}$ gaben $0,4080 \mathrm{~g}$ Chlor- und Bromsilber. $0,362 \mathrm{~g}$ des letzteren verloren beim Erhitzen im Chlorstrom 0,0401 g.

Gef.

I

Au 33,9

$\mathrm{Br}$

$\mathrm{Cl}-$
II

27,6?

18,24

Ber. für $\mathrm{C}_{4} \mathrm{H}_{8} \mathrm{~N}_{8} \mathrm{~S} \mathrm{Br}_{2} \mathrm{Au} \mathrm{Cl}_{8}$

Meine Verbindung war also als ein einfaches Additionsprodukt von Thiosinamindibromid und Goldchlorid aufzufassen. Jedoch verläuft der Prozels nicht immer in derselben Weise. Ein anderes, gleichzeitig dargestelltes Doppelsalz unterschied sich von dem obigen durch seine dunkelrote Farbe. Ein Teil desselben wurde aus Alkohol umkrystallisiert, wobei es in dicken, stahlglänzenden Nadeln, welche zu kreisförmigen Büscheln vereinigt waren, resultierte. Fine Goldbestimmung ergab 29,4 Proz. Au, genau entsprechend den Angaben Maly's. War nun die von Ma $\mathrm{l}$ y angegebene Umsetzungsgleichung richtig, so mufste in den Mutterlaugen des zweiten Präparates noch eine grolse Menge des Thiosinaminbromochlorids enthalten sein, da das gebildete Goldbromid nicht hinreicht, um alles in das Goldsalz überzuführen. Um dies zu konstatieren, fällte ich aus den Mutterlaugen das Gold mit Schwefelwasserstoff aus und dampfte das Filtrat auf ein kleines Volumen ein. Nach einiger Zeit krystallisierten schöne, grofse, rautenförmige, farblose Tafeln aus, deren Schmelzpunkt bei 128,50 lag ( $M$ a I $\mathrm{y}^{\prime}$ 's Chlorobromid 129-130\%). Ein daraus dargestelltes Goldsalz war von rotgelber Farbe.

$0,3384 \mathbf{g}$ gaben $0,1239 \mathrm{~g}$ Gold.

Gefunden: $\quad$ Berechnet für $\mathrm{C}_{4} \mathrm{H}_{8} \mathrm{~N}_{2} \mathrm{~S} \mathrm{Br} . \mathrm{Cl}, \mathrm{Au} \mathrm{Cl} \mathrm{Cl}_{3}$ Au 36,61 36,7 .

Aus Vorstehendem erhellt auch, dals bei der Darstellung dieses zweiten Präparates das Goldchlorid in zu geringer Menge zugesetzt worden war, da sich ja sonst noch das Goldsalz des Bromochlorids hätte ausscheiden müssen. Dieser Umstand läfst darauf schliefsen, dafs dem Goldsalz, je nach den Darstellungsbedingungen, eine andere Zusammensetzung zukommen wird. Meine noch weiter angestellten Versuche haben nun ergeben, dafs man es hier mit einem Beispiel der Massenwirkung zu thun hat. Ist das Goldchlorid im Ueberschufs, so wird das Präparat chlorreich und arm an Brom, während bei einem Ueberschufs des Thiosinaminbromids das Umgekehrte eintritt. 
Zum Beweise dafür mögen folgende Beispiele dienen:

1. Eine wässrige Lösung des Bromids wurde mit einer ungenügenden Menge Goldchlorid versetzt. Es entstand ein dunkelroter Niederschlag.

$0,2535 \mathrm{~g}$ hinterlielsen beim Glühen $0,0709 \mathrm{~g} \mathrm{Au}$.
Gefurden :
Berechnet für $\mathrm{C}_{4} \mathrm{H}_{8} \mathrm{~N}_{2} \mathrm{~S} \mathrm{Br}_{2} \mathrm{Au} \mathrm{Br} r_{3}$

Au 27,96

27,60 .

Das Präparat enthielt also nahezu nur Brom.

2. Eine gleiche Lösung wurde mit etwas mehr Goldchlorid versetzt. Es entstand wiederum ein dunkelroter Niederschlag, den ich aus Alkohol umkrystallisierte. Stahlglänzende Nadeln.

$0,1882 \mathrm{~g}$ hinterlielsen beim Glihen $0,0548 \mathrm{~g}$ Au.
Gefunden :
Berechnet für $\mathrm{C}_{4} \mathrm{H}_{8} \mathrm{~N}_{2} \mathrm{~S} \mathrm{Br} \mathrm{Cl}$. $\mathrm{Au} \mathrm{Br} 3$

Au 2914

3. Eine wässrige Lösung des Thiosinaminbromids mit Goldchlorid versetzt, bis keine Fällung mehr eintrat, schied ein ziegelrotas Krystallpulver ab.

a) $0,5072 \mathrm{~g}$ lieferten beim Glühen $0,1671 \mathrm{~g} \mathrm{Au}$.

Dasselbe, aus Alkohol umkrystallisiert, resultierte in rubinroten Krystallen.

b) $0,1898 \mathrm{~g}$ hinterlielsen beim Glühen $0,0617 \mathrm{~g} \mathrm{Au}$.

Gafunden :

I. II.

Au $\quad 32,94 \quad 32,5 \mathrm{I}$
Keine einheitliche Formel. Gemisch aus: $\mathrm{C}_{4} \mathrm{H}_{8} \mathrm{~N}_{2} \mathrm{~S} \mathrm{Br} \mathrm{Cl} . \mathrm{Au} \mathrm{Br}$

und

Ein ähnliches Gemisch dürfte das von Falke dargeste'Ite und analysierte Präparat gewesen sein.

4. Eine wässrige Lösung mit Goldchlorid im Ueberschuls verset/t, schied sofort einen gelbroten krystallinischen Niederschlag ab. Derselbe wurde sofort abgesaugt und nacb dem Trocknen analysiert.

a) $0,4592 \mathrm{~g}$ ergalen beim Glühen $0,1557 \mathrm{~g}$ Au.

Dasselbe, aus Alkohol umkrystallisiert, resultierte in orangeroten, gut ausgebildeten $K_{r}$ ystallen.

b) $0,1283 \mathrm{~g}$ hinterliefsen $0,0434 \mathrm{~g}$ Gold.

Ein anderes, ebenfalls in derselben Weise dargestelltes, aus Alkohol umkrystallisiertes Präparat, war einen Stich röter gefärbt.

c) 0,2594 $\mathrm{g}$ lieferten beim Glühen $0,087 \mathrm{~g}$ Gold.

\begin{tabular}{|c|c|c|c|}
\hline & & II & $\mathrm{B} \approx \mathrm{r}$. für \\
\hline $\mathrm{Au} 33,91$ & 33,83 & 33,54 & 33,94 . \\
\hline
\end{tabular}

5. Eine wässrige Lösung wurde auf einmal mit einem starken Ueberschuis von Goldchlorid versetzt. Der entstandene, gelbrote Niederschlag blieb zwei Tage mit den Mutterlaugen in Berührung. 
Der Niederschlag sah nach dem Absaugen rötlich-gelb aus; die Mutterlauge war blutrot gefärbt.

$0,1685 \mathrm{~g}$ des getrockneten Präparates hinterlielsen beim Glühen $0,0600 \mathrm{~g} \mathrm{Au}$.

$$
\begin{array}{cc}
\text { Gef. } & \text { Ber. für } \mathrm{C}_{4} \mathrm{H}_{8} \mathrm{~N}_{2} \mathrm{SBrCl} . \mathrm{Au} \mathrm{Cl}_{3} \\
\mathrm{Au} 35,61 & 36,7 .
\end{array}
$$

Das Präparat war also als ein Gemisch der Goldsalze des Dibromids und des Bromochlorids aufzufassen.

Fassen wir diese Resultate noch einmal kurz zusammen: Der Goldgehalt schwankt in den einzelnen Präparaten zwischen 27,96 und 35,61 Proz. Daraus folgen nun Verbindungen, die, ausgehend vom Thiosinamindibromid-Goldbromid allmählich Brom gegen Chlor austauschen, bis endlich nahezu reines ThiosinaminbromochloridGoldchlorid entsteht. Eine weitere Ersetzung von Brom durch Chlor ist, wie schon oben erwähnt, nicht möglich, da das eine noch übrige Bromatom organisch gebunden ist.

Einwirkung von Pikrinsäureauf

Thiosinaminbromid.

Versetzt man eine wässrige Thiosinaminbromidlösung mit wässriger Pikrinsäure, so erhält man einen voluminösen Niederschlag, der sich beim Erwärmen vollständig klar auflöst. Beim Erkalten krystallisiert das Pikrat in gelben, durchsichtigen Nadeln von ca $5 \mathrm{~mm}$ Länge. Ihr Schmelzpunkt liegt bei $184-185^{\circ}$.

Das bei $100^{\circ}$ getrocknete und zerriebene Salz unterwarf ich zur Bestimmung des Bromgehaltes, einer Analyse nach Carius.

$0,1721 \mathrm{~g}$ lieferten dabei $0,0755 \mathrm{~g}$ Ag Br.

Gef.

Br. $\quad 18,67$
Ber. tür $\mathrm{C}_{4} \mathrm{H}_{7} \mathrm{~N}_{2} \mathrm{SBr} . \mathrm{C}_{6} \mathrm{H}_{2}\left(\mathrm{NO}_{2}\right)_{3} \mathrm{OH}$

$$
18,86 \text {. }
$$

Einwirkung von Chlorsilber auf Thiosinaminbromid.

Durch Einwirkung von Chlorsilber auf Thiosinaminbronid erhält man, wie M a ly und F a $1 \mathrm{k}$ e übereinstimmend berichten, das Thiosinaminbromochlorid. Ihre Angaben über den Schmelzpunkt differieren jedoch so erheblich, dafs ich zur Feststellung desselben die Verbindung noch einmal darstellte.

Das aus den Mutterlaugen des Einwirkungsproduktes von Goldchlorid auf Thiosinaminbromid erhaltene Bromochlorid schmolz, wie schon erwähnt, bei 128,50 . Das nach $\mathrm{M}$ a ly, mit Hilfe von 
Chlorsilber, dargestellte Präparat war eine in Wasser und Alkohol leicht lösliche Krystallmasse ohne deutlich ausgebildete Einzelkrystalle. Solche konnten auch aus Alkohol nicht erhalten werden, wohl aber aus einem Gemisch von Alkohol und Essigäther. In reinem Essigäther ist die Verbindung unlöslich.

Der Schmelzpunkt des ursprünglich auskrystallisierten Salzes lag bei $128,5^{\circ}$, der des aus Essigäther-Alkohol umkrystallisierten bei 128,5 -129,5. Ich fand somit die Angaben Maly's, entgegen denen von Falke, bestätigt. Falke giebt nämlich $118-120^{\circ}$ an.

Einwirkung von Silberoxydaufdas Thiosinamin. bromid.

Die Angaben Falke's über die Einwirkung von einem Molekül Silberoxyd auf 2 Moleküle Thiosinaminbromid kann ich im allgemeinen nur bestätigen. Die entstehende treie Base ist nicht krystallisierbar, sondern behält selbst nach monatelangem Stehen über Schwefelsäure ihre sirupöse Beschaffenheit. Mit Salzsäure verbindet sie sich leicht zu Bromochlorid; so erstarrte dieselbe bei mir nach einiger Zeit zu weilsen, strahlig anceordneten Krystallmassen, als ich sie zugleich mit einer salzsäurehaltigen Flüssigkeit in Exsiccator aufbewahrte. Diese von der anhaftenden fi'eien Base durch Pressen zwischen Thonplatten befreiten Krystalle schmolzen bei 128,5-129 . Beim Titrieren mit Silbernitrat wiesen sie einen Gehalt von 15,22 Proz. Cl auf, während 15,33 Proz. berechnet sind. Die Angabe F a lke's, dafs die Base beim Kochen mit gelbern Quecksilberoxyd ein quecksilberhaltiges Filtrat gegeben habe, welches er mit Zinnchlorür nachgewiesen habe, bedarf insofern einer Berichtigung, als das Thiosinamin selbst sowohl, als auch die freie Base für sich schon mit Zinnchlorür weifse Niederschläge liefert.

Bei der Einwirkung von überschüssigem Silberoxyd machte F a lke die Wahrnehmung, dafs selbiges sich beim Kochen am Rückflufskühler dunkler färbte und mit dem aus dem Thiosinaminbromid entstandenen Körper eine wasserunlösliche Doppelverbindung eingeht. Letztere versuchte er durch Kochen mit Salzaäure zu zerlegen. Er erhielt dadurch einen sirupartigen Rückstand, der nicht krystallisationsfähig war, und von dem er deshalb das Platinsalz darstellte. Die von demselben ausgetührten Analysen ergaben Resultate, 
die sich anf eine Einwirkung des Silberoxyds analog überschüssigem Silbernitrat nicht vereinigen lielsen, ebensowenig auf eine blofse Abspaltung von Bromwasserstoff schliefsen lielsen. Dem mufs ich noch hinzufügen, dafs, selbst durch Kochen mit Salzsäure, die Zerlegung der Silberoxydverbindung nur äulserst schwierig und unvollkommen erfolgt. Die Ausbeute steht in keinem Verhältnis zur angewandten Menge. Auf Zusatz von Ammoniak erfolgt die Abscheidung eines voluminösen, schwach bräunlich gefärbten Körpers.

Einen Teil führte ich, wie $\mathrm{Falk} \theta$, in das Platinsalz über. Die von mir gefundenen Werte stimmen annähernd mit denen F a lke's überein.

1. $0,1250 \mathrm{~g}$ hinterliefsen beim Glühen $0,0357 \mathrm{~g}$ Pt.

2. $0,1198 \mathrm{~g}$ lieferten $0,0724 \mathrm{~g} \mathrm{CO}_{2}$ und $0,0331 \mathrm{~g} \mathrm{H}_{2} \mathrm{O}$.

\begin{tabular}{ccc}
\multicolumn{2}{c}{ Gef. } & Falke fand \\
I & II & \\
Pt 28,56 & - & 28,2 \\
C - & 16,5 & $17,3-17,7$ \\
H $-~$ & 3,07 & $2,9-3,2$.
\end{tabular}

Einen zwoiten, möglicl:st :on Salzsäure durch Eindampfen befreiten Teil versetzte ich, nach dem Verdünnen mit Wasser und Filtrieren, mit stark ammoniakalischer Chlorsilberlösung. Es entstand dadurch ein feiner, voluminöser, schwach bräunlich gefärbter Niederschlag, der nach dem Absaugen und Auswaschen mit Ammoniak über Schwefelsäure getrocknet wurde. Dabei schrumpfte er zu einer gummiartigen Masse zusammen, die infolge Zersetzung braunschwarz gefärbt war.

$0,1314 \mathrm{~g}$ dieser Verbindung wurden mit Salpetersäure gekocht; das dabei resultierende Chlorsilber betrug $0,0487 \mathrm{~g}$. Im Filtrat erfolgte auf Zusatz von Salzsäure eine weitere Abscheidung von Chlorsilber im Gewicht von $0,033 \mathrm{~g}$.

$$
\begin{aligned}
& \text { Gefunden. } \\
& \text { Cl } 9,18 \\
& \text { Ag } 46,79 .
\end{aligned}
$$

Diese Werte lassen ebenfalls keinen Schlufs über die Natur des Körpers zu und sind überhaupt ohne Interesse, da die ursprüngliche Verbindung effenbar zersetzt war.

Den geringen Rest des durch Auskochen mit Salzäure erhaltenon, sirupartigen Körpers versetzte ich mit Salpetersäurehydrat. Als die Einwirkung desselben aufgehört, verdünnte ich mit Wasser 
und prüfte mit Chlorbaryum auf Schwefelsäure. Da selbst nach längerer Zeit keine Trübung eintrat, war hierdurch lewiesen, dals das Silberoxyd im Ueherschuls entschwefelnd auf das Thiosinaminbromid gewirkt hatte. In welcher Weise jrdoch diese Einwirkung stattgefunden, lärst sich bei der Schwierigkeit der Reindarstellung des Reaktionsproduktes nicht nachweisen, vielleicht hat noch eine Oxydation mitgespielt. Der Umstand jedoch, dals im Platinsalz auf 1 Atom Platin 10 Atome Koblenstoff kommen, lälst vermuten, dafs das Einwirkungaprodukt kein einheitliches sei. -

Thiosinaminjodid.

Durch Einwirkung alkoholischer Jodlösung auf eine Lösung von Thiosinamin in Alkohol entsteht nach $\mathrm{Mal} \mathrm{y}^{\mathbf{1}}$ ) ein Thiosinaminodid genau in derselben Weise, wie durch Einwirkung von Brom ein Bromid geljildet wird. Es findet dabei jedoch nur eine sehr geringe Erwärmung statt. Beim freiwilligen Verdunsten scheiden sich harte, schwach gelblich gefärbte, durchsichtige Krystalle aus, deren Schmelzpunkt bei $130,5^{0}$ liegt, wobei gleichzeitig eine Zersetzung der Verbindung vor sich geht, während M a ly als solchen $90^{\circ}$ angiebt.

Gegen Silbernitrat verhält sich diese Verbindung etwas anders, als das Bromid. Denn wäbrend Silbernitrat (nach $\mathrm{Falk} \theta$ ) aus dem Bromid nur beim Kochen sämtliches Brom zu eliminieren vermag, bei gewöhnlicher Temperatur aber nur e in Atom, entfernt dasselbe aus dem Jodid bereits in der Kälte sämtliches Jod. Dafs aber auch die beiden Jodatome sich verschieden gegen Silbernitrat verhalt $n$, kann man aus einer Jodbestimmung durch Titration erkennen. Eine direkte Analyse mit 1/10 N. Silberlösung, unter Zusatz von Kaliumchromat als Indikator, liefert allerdings unbrauchbare Resultate, da der Farbenumschlag nicht genau zu sehen ist. Hingegen führt eine Silber-Restbestimmung mit Rhodanammonium zu guten Resultaten, wenn sofort nach Zusatz der überschüssigen Silberlösung der Ueberschuls mit Rhodanammoniumlösung, unter Zusatz von Eisenalaun als Indikator, zurücktitriert wird. Auf diese Weise wird nur ein Atom Jod als Jodsilber gefällt. Lälst man das

1) Ztsch. f. Chemie 1869, 258. 
Silbernitrat längere Zeit einwirken, so eliminiert es auch das zweite Jodatom.

Die von der Verbindung ausgeführten Analysen gaben folgende Resultate :

1. $0,4627 \mathrm{~g}$ lieferten $0,2190 \mathrm{~g} \mathrm{CO}_{2}$ und $0,0957 \mathrm{~g} \mathrm{H}_{2} \mathrm{O}$.

2. $0,388 \mathrm{~g}$ gaben nach Carius $), 2441 \mathrm{~g} \mathrm{BaSO}_{4}$.

3. $0.5831 \mathrm{~g}$ mit Silbernitrat in der Kälte versetzt und nach 24 Stunden abfiltriert, lieferten $0,7383 \mathrm{~g} \mathrm{AgJ}$.

4. $0,4693 \mathrm{~g}$ mit überschüssigem Silbernitrat, unter Zusatz einiger Tropfen Salpetersäure, gekocht, schieden 0,6110 g Jodsilber ab.

5. $0,1304 \mathbf{g}$, in der oben angegebenen Weise titriert, verbrauchten $3,55 \mathrm{ccm} 1 / 10 \mathrm{~N}$ Silberlösung.

\section{Gefunden :}

I II III

12.92

2,31

$-\quad 8,64$
IV

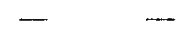

$\mathrm{H}$

8

$\mathrm{J}$

J als
-

\section{V} $\nabla$.

Berechnet für $\mathrm{C}_{4} \mathrm{H}_{8} \mathrm{~N}_{2} \mathrm{~S} \cdot \mathrm{J}_{2}$ :

Mit Gold- und Platinchlorid giebt las Jodid keine einheitlichen Verbindungen, da sich auf Zusatz dieser Agentien sofort Jod abscheidet. Hingegen erhält man auf Zusatz von Pikrinsäure, Phosphormolybdän- und Phosphorwolframsäure kräftige Niederschläge, ebenso auch mit den Doppeljodiden. Von diesen benutzte ich ảas Pikrat zur weiteren Untersuchung. Der amorphe, kräftige Niederschlag, der sich zunächst beim Zusatz wässriger Pikrinsäure bildet, löst sich beim Kochen wieder klar auf. Beim Erkalten resultiert die Verbindung in prachtvoll glänzenden, wasserfreien, durchsichtigen Krystallblättchen, welche direkt analysenrein sind.

Der Schmelzpunkt liegt bei 178-1790.

$0,276 \mathrm{~g}$ der bei $100^{\circ}$ getrockneten Substanz, nach Carius mit Silbernitrat und Salpetersäurehydrat 3 Stunden auf $180^{\circ}$ erhitzt, lieferten $0,1378 \mathrm{~g} \mathrm{AgJ}$.

Gef. Ber. für $\mathrm{C}_{4} \mathrm{H}_{7} \mathrm{~N}_{2} \mathrm{SJ} . \mathrm{C}_{6} \mathrm{H}_{2}\left(\mathrm{NO}_{2}\right)_{3} \mathrm{OH}$

J 26,94 26.96 .

Thiosinaminjodochlorid.

Durch Behandeln von Thiosinaminjodidlösung mit Chlorsilber geht dasselbe, wie bereits $\mathrm{Maly}$ angiebt, durch Austausch eines Atomes Jod gegen Chlor, in das Thiosinaminjodochlorid über; das- 
selbe resultiert aus dem eingeengten Filtrat in aufserordentlich leicht in Alkohol und Wasser löslichen, aber gut ausgebildeten Krystallen, von schwach gelblicher Farbung. Der Schmelzpunkt liegt höher, als der des Jodids selbst, numlich bei 132-1330, während der Schmelzpunkt des entsprechenden Bromochlorids etwa um $10^{\circ}$ niedriger, als der des Bromids ist.

Der Chlorgehalt des Thiosinaminjodochlorids läst sich in der für das Jodid angegebenen Weige leicht und scharf durch titrimetrische Restbestimmung ermitteln.

Zur Sättigung von $0,0705 \mathrm{~g}$ des Salzes wurden einmal $24,3 \mathrm{ccm}$ einer $1 / 100 \mathrm{~N}$ Silberlosung verbraucht, das andere $\mathrm{mal} 24,5 \mathrm{ccm}$.

Gefunden :

I II

Cl $\quad 12,57 \quad 12,68$
Berechnet fur

$\mathrm{C}_{4} \mathrm{H}_{7} \mathrm{JN}_{2} \mathrm{~S} . \mathrm{H} \mathrm{Cl}$.

12.75 .

Die mit Salzsäure angesănerte Lösung des Thiosinaminjodochlorids giebt mit Gold- und Platinchlorid krystallinische Niederschläge, die, nach dem Trocknen uther Schwefelsăure, direkt analysiert werden konnten.

$0.35 i 6 g$ des Goldsalzes zersetzten sich bereits im Trockenschrank durch Abgabe von Jod; bis zum konstanten Gowicht gegluht, hinterliefsen sie 0,121 Gold. Beim Bestimmen des Schmelzpunktes im Kapillarrohr fand ich denselben als bei $113^{\circ}$ liegend.

Gefunden: Berechnet für $\mathrm{C}_{4} \mathrm{H}_{7} \mathrm{JN}_{2} \mathrm{~S} \cdot \mathrm{HCl} \cdot \mathrm{Au} \mathrm{Cl} \mathrm{Cl}_{3}$

Au $33,8 \quad 33,8$

Das Platinsalz war bei 1000 beständig und erwies sich, wie das Goldsalz, als wasserfrei.

Gefunden: Berechnet fur $\left[\mathrm{C}_{4} \mathrm{H}_{7} \mathrm{JN2S} \cdot \mathrm{HCl}_{2} \mathrm{Pt} \mathrm{Cl}_{4}\right.$

Pt. 22,00

22,02

Einwirkung von üborschägsigem Silbernitrat a u $\mathrm{Th}$ iosinaminjodid.

Ueberschüssiges Silbernitrat wirkt, wie ich bereits oben angegeben habe, auf das Thiosinaminjodid. im Gegensatz zum Bromid, in der Käle und Wärme in gleicher Weise, indem es alles Jod entzieht. Bei analoger Konstitution des Jodids mufs die dabei entstehende Verbindung jentisch mit der von Falke durch Erhitzen des Bromids mit uberschulssigem Silbernitrat erhaltenen sein. Um dies zu konstatieren, versetzte ich $10 \mathrm{~g}$ des Jodids mit einer berechneten Menge Silbernitrat, so dafs auf ein Molekül des Salzes etwas mehr als 2 Molekule Silbernitrat kamen. Nach mehrtägigem 
Einwirken in der Kalte wurde die klare Flüssigkeit vom ausgeschiedenen Jodsilber abfiltriert, und im Filtrat der geringe Ueberschufs an Silbernitrat mit Salzsäure entfernt.

Einen kleinen Teil des Filtrates versetzte ich mit Pikrinsăure; dieselbe verursachte einen flockigen Niederschlag, der sich beim Erwärmen löste, beim Er':alten krystallinisch wieder abschied.

Einen anderen, grölseren Teil versetzte ich mit überschüssigem Platinchlorid. Da direkt keine Ausscheidung stattfand, liels ich die Lösung über Schwefelsäure verdunsten; dabei krystallisierte das Platinsalz nach einiger Zeit in schönen, gelbroten Rhomboëdern aus. Aus den Mutterlangen krystallisierten noch weitere Mengen von demselben Aellfseren, aufserdem aber noch gelb gefarbte, undurchsichtige, quadratische Taf $e \ln$, letztere jedoch in so geringer Menge, dafs ich von ihnen keine Bestimnung ausführen konnte. Erstere erwiesen sich beim Trocknen bei $100^{\circ}$ als wasserfrei.

$0,2664 \mathrm{~g}$ hinterliefsen beim Gluhen $0,0775 \mathrm{~g} \mathrm{Pt}$.

Gefunden : Berechnet für $\left[\mathrm{C}_{4} \mathrm{H}_{7} \mathrm{OHN2} \mathrm{S} \cdot \mathrm{H} \mathrm{Cl}_{2} \mathrm{Pt} \mathrm{Cl}_{4}\right.$

Pt. 29,15 28,88

Den Rest obigen Filtrates führte ich in das Goldsalz uber. Goldchlorid veranlafste zunächst in der salzsauren Flüssigkeit eine milchige Trübung, die jedoch schon bei gelindem Erwärmen (etwa auf $35^{\circ}$ ) einer klaren Lösung wich. Beim Erkalten derselben schied sich nach wenigen Stuniten ein öliges, rotes Liquidum ab, welches durch Reiben mit einem Glasstab nicht zum Krystallisieren gebracht werden konnte. Ich löste es deshalb noch einmal, unter Zusatz von etwas Alkohol und Salzsaure, auf und liess die Lösung auf einem flachen Uhrglas langsam verdunsten. Wiederum schied sich die Hauptmenge als öliges Liquidum ab, während nur am Rande kleine, gelbe Nadeln krystallisierten. Als ich von letzteren einige Kryställchen in das ölige Liquidum brachte, erstarrte dasselbe zu gelben Krystallmassen. Dieselben erwiesen sich nach dem Abspritzen mit Alkohol ebenfalls als wasserfrei.
$0,436 \mathrm{~g}$ hinterliefsen beim Glithen $0,1810 \mathrm{~g} \mathrm{Au}$. Gef.
Au 41,51
Ber. fur $\mathrm{C}_{4} \mathrm{H}_{7} \mathrm{OH} \mathrm{\textrm {N } _ { 2 } \mathrm { SH } \mathrm { Cl } \text { . Au Cl}}$ 41,68 .

Diese Werte lassen es keinem Zweifel unterliegen, dals das Einwirkungsprodukt von uberschtissigem Silbernitrat auf Th:usinaminjodid identisch ist mit dem, welches Thiosinaminbromid bei gleicher 
Behandlungsweise liefert. Daraus folgt, dafs beide Körper dieselbe Konstitution besitzen müssen, dafs also auch die Jodverbindung von einer unsymmetrischen Formel des Thiosinamins durch Ringbildung und Jodwasserstoffabspaltung abzuleiten ist.

Darstellung derfreien Basedes Thiosinaminjodids mittels Silberoxyd.

Bei dem völlig gleichen Verhalten des Jodids und Bromids des Thiosinamins war zu erwarten, dafs ersteres, ebenso wie letzteres, durch Silberoxyd in die freie Base übergeführt werden konnte. Ich versetzte daher $4 \mathrm{~g}$ Thiosinaminjodid mit einer derartig berechneten Menge frischgefällten Silberoxyds, dafs auf 2 Moleküle Thiosinaminjodid e in Molekül Silberoxyd kam. Es spaltete sich sofort das als Jodwasserstoff vorhandene Jodatom ab, und die Flüssigkeit nahm stark alkalische Reaktion an. Beim Verdunsten des bitter und etwas schrumpfend schmeckenden Filtrates resultierte eine gummiartige Masse, die sich nur zum Teil wieder in Wasser oder Alkohol löste, während der grölste Teil, der wahrscheinlich infolge Polymerisation eine Molekularveränderung erlitten hatte, ungelöst blieb. Das Filtrat nahm, bein nochmaligen Verdunsten über Schwefelsäure, dieselbe Beschaffenheit an.

In dem über Schwefelsäure getrockneten Präparate bestimmte ich den Gehalt an Jod und Schwefel nach dem Verfahren von Carius.

$0,1763 \mathrm{~g}$ lieferten dabei $0,1692 \mathrm{~g} \mathrm{Ag} \mathbf{J}$ und $0,176 \mathrm{~g} \mathrm{Ba} \mathrm{SO}$.
Gef.
J $5 ! .86$
Ber. für $\mathrm{C}_{4} \mathrm{H}_{7} \mathrm{JN}_{2} \mathrm{~S}$
S 13,71
52,45
13,22 .

Der zu hohe Befund an Schwefel und der zu geringe an Jod dürfte seine Erklärung darin finden, dals das im geringen Ueberschuls angewandte Silberoxyd auch einen geringen Teil des organisch gebundenen Jods eliminiert hat.

M a ly hat für die in entsprechender Weise aus dem Bromid dargestellte, freie Base die Formel

$$
\mathrm{C}_{4} \mathrm{H}_{7} \mathrm{Br} \mathrm{N} \mathrm{N}_{2} \mathrm{~S} \text { OH }
$$

aufgestellt, in der Annahme, dafs 1 Atom Brom gegen die Hydroxylgruppe des feuchten Silberoxyds ausgetauscht sei. F a 1 ke hat indessen durch das Verhalten der freien Base gegen Jodäthyl and durch die Analogie der von Andreasch aus dem Dibrom- 
propylharnstoff dargestellten Base, welche keine OH Gruppe enthält, das Unwahrscheinliche der $\mathbf{M}$ a ly'schen Formel nachgewiesen. Durch meine Analyse wird $\mathrm{F}$ alk $\theta^{\prime} \mathrm{s}$ Ansicht bestätigt; denn die Formel $\mathrm{C}_{4} \mathrm{H}_{7} \mathrm{JN}_{2} \mathrm{SOH}$ würde nur 44,78 Proz. J und 12,35 Proz. S verlangen, während die von mir ermittelten Werte hinreichend auf die Formel $\mathrm{C}_{4} \mathrm{H}_{7} \mathrm{JN}_{2} \mathrm{~S}$ passen.

Einwirkung von Chlor auf Thiosinamin.

Der Umstand, dafs das Thiosinaminbromid und -jodid schon lange bekannt sind, während sich von einer entsprechenden Chlorverbindung keine Angaben in der Litteratur vorfinden, lälst vermuten, dafs Chlor nicht in der nämlichen Weise, wie Brom und Jod, auf eine alkoholische Thiosinaminlösung einwirken, sondern, vermöge seiner viel stärker oxydierend wirkenden Eigenschaften, eine tiefer greifende Zersetzung herbeiführen wird. Meine Vermutung sah ich durch das Experiment bestätigt.

In eine alkoholische, mäfsig konzentrierte Thiosinaminlösung leitete ich unter Abkühlung einen langsamen Chlorstrom, bis letzterer nicht mehr absorbiert wurde. Das Reaktionsgemisch wurde bei mälsiger Wärme eingeengt und über Schwefelsäur e zur Krystallisation gestellt. Es fand jedoch keine Abscheidung von Krystallen statt, und es verblieb nur ein gelblicher Sirup, der sich auf Zusatz von Wasser schwach träbte. Da nun das Thiosinaminbromid und -jodid in Aether unlöslich sind, so ist diese Eigenschaft auch für die entsprechende Chlorverbindung vorauszusetzen; es konnte sich somit hierdurch eine Möglichkeit bieten, das eventuell gebildete Thiosinaminchlorid krystallisiert zu erhalten. Ich überschichtete demgemäls eine alkoholische Lösung des Einwirkungsproduktes in einer Flasche mit Aether. Nach einigen Wochen war die alkoholische Schicht bis auf eine geringe ölige Flüssigkeit verschwunden. Von letzterer nahm ich mit einer Pipette einige Tropfen heraus, löste sie in salzsäurehaltigem Wasser und setzte zu je einer Probe Gold- und Platinchlorid zu. Da selbst nach längerem Stehen weder ein Niederschlag, noch eine Abscheidung von Krystallen zu bemerken war, die entsprechende Brom- und Jodverbindung aber mit diesen Agentien gut charakterisierte, schwer lösliche Verbindungen lieferte, so war es unwahrscheinlich, dals sich Thiosinaminchlorid unter obigen Be- 
dingungen überhaupt gebildet hatte. Unverändertes Thiosinamin konnte die ölige Flüssigkeit jedoch auch nicht enthalten, da dieses einerseits in Aether löslich ist, andererseits mit Platinchlorid eine unlösliche Doppelverbindung liefert.

Ich versuchte nun zu dem Thiosinaminchlorid auf andere Weise zu gelangen, nämlich durch direkte Einwirkung von trockenem Chlor auf gepulvertes Thiosinamin. Zu diesem Zweck breitete ich zerriebenes Thiosinamin in einer etwa $60 \mathrm{~cm}$ langen Glasröhre in dünner Schicht aus und liefs nun einen langsamen Chlorstrom darüber streichen.

Dafs Chlor auf Thiosinamin überhaupt einwirkte, konnte ich daran erkennen, dafs an der Eintrittastelle des Chlors das Thiosinamin allmählich eine zähe und durchsichtige Beschaffenheit annahm, während gleichzeitig eine lebhafte Erwärmung zu bemerken war.

Bei fortgesetztem Durchleiten traten am andern Ende des Rohres saure Dämpfe aus, ein Zeichen, dafs Chlor nicht blos addierend auf die Allylgruppe, sondern auch substituierend wirkte. Nach etwa acht Stunden unterbrach ich den Chlorstrom; das Reaktionsprodukt löste ich in wenig Alkohol auf und versetzte es mit Aether im Ueberschuls; dabei entstand eine milchige Trübung, welche allmählich verschwand, während sich eine zähe, durchsichtige Masse zu Boden setzte. Letztere, welche eventuell Thiosinaminchlorid enthalten konnte, reinigte ich durch wiederholtes Auflösen in Alkohol und Fällen mit Aether. Als ich annehmen konnte, dafs alles unveränderte Thiosinamin auf diese Weise entfernt sei, versetzte ich den Rückstand mit Wasser, womit er eine etwas trübe Lösung gab, die aber durch Filtrieren geklärt werden konnte. Dieselbe lieferte die gleichen Reaktionen, wie das Brom- and Jodadditionsprodukt. Zur Analyse benutzte ich das Platinsalz und das Pikrat.

Das orangefarbene Platinsalz war amorph. 0,4937 $\mathbf{g}$ des lufttrocknen Salzes verloren bei $100^{\circ} 0,0194 \mathrm{~g} \mathrm{H}_{2} \mathrm{O}$, welches wohl nur hygroskopische Feuchtigkeit sein mochte, und hinterliefsen beim Glühen 0,137 g Platin.

Gefunden

Pt 1. für luftrockne Substanz:

27,75

2. für die bei $100^{\circ}$ getrocknete: 28,88
Berechnet für $\left[\mathrm{C}_{4} \mathrm{H}_{8} \mathrm{~N}_{2} \mathrm{SCl}_{2}\right]_{2} \mathrm{Pt} \mathrm{Cl}_{4}$. $27,3 i$ 
Wenn der für die lufttrockene Substanz gefundene Wert auch einigermafsen mit dem berechneten übereinstimmt, und der Verlust bei $100^{\circ}$ durch Abgabe von etwas Chlor verursacht sein könnte, lälst mich doch die Analyse des Pikrates darauf schliefsen, dafs obiges Platinsalz kein einheitliches gewesen sei, sondern ein Gemisch aus dem Platindoppelsalz des Thiosinaminchlorids und dem des Propylen- $\psi$-Thioharnstoffs vorstellte. Letzteres konnte sich leicht bilden, da ja, wie oben erwähnt, bei dem Leiten von Chlor über Thiosinamin sich reichliche Mengen von Salzsäure bildeten, die i hrerseits auf eine neue Menge des Thiosinamins, in der von Gabriel angegebenen Weise, umlagernd wirken konnten.

Das Pikrat, dessen Schmelzpunkt bei $173-174^{\circ} \mathrm{lag}$, lieferte bei einer Chlorbestimmung nach Carius folgendes Resultat: $0,1873 \mathbf{g}$ ergaben $0,0230 \mathrm{~g} \mathrm{Ag} \mathrm{Cl}$.

Gef. Berechnet für $\mathrm{C}_{4} \mathrm{H}_{7} \mathrm{~N}_{2} \mathrm{~S} \mathrm{Cl}, \mathrm{C}_{6} \mathrm{H}_{2}\left(\mathrm{NO}_{2}\right)_{3} \mathrm{OH}$

$$
\text { Cl. } 3,04 \quad 9,35 \text {. }
$$

Leider ist es mir nicht gelungen, das Thiosinaminchlorid $r$ in darzustellen. Immerhin aber geht aus Obigem hervor, dals unter geeigneten Verhältnissen dasselbe neben anderen Körpern entsteht.

Versuche zur Ermittelung der Konstitution des Thiosinaminbromids resp. -jodids.

Nachdem ich somit durch Hervorhebung der gemeinschaftlichen Eigenschaften des Thiosinaminbromids und -jodids nachgewiesen habe, dafs beiden Körpern dieselbe Konstitution zukommen mufs, erübrigt es nun noch, diese selbst zu erforschen. Die Wege, welche sich dazu bieten, liegen, wie schon kurz angedeutet, in folgendem.

Da sich das Thiosinaminbromid resp. -jodid von der unsymmetrischen Formel ableiten, so kann denselben nur eine der Formeln

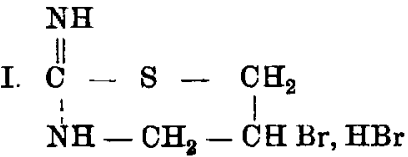
$\mathrm{NH}$<smiles>CC(Br)SC(C)CCBr</smiles>

odir eine der beiden vorher sub III und IV angeführten, zukommen. Die letzteren beiden mögen aber zunächst, unter Berücksichtigung des von F a l ke konstatierten Imidcharakters der fraglichen Verbindung, als wenig wahrscheinlich aulser Acht gelassen bleiben. 
Die zweite Formel, welche einen bromwasserstoffsauren $\beta$ Brommethyläthylen $\psi$ Thioharnstoff entsprechen würde, mufste den Vorzug verdienen, wenn es gelang, diese Verbindung durch naszierenden Wasserstoff, aus saurer oder alkalischer Quelle, zu dem $\beta$ Methyläthylen $\psi$ Thioharnstoff $\mathrm{G}$ a b r i $\theta \mathrm{l}$ 's, den derselbe der Kürze wegen Propylen $\psi$ Thioharnstoff nennt, zu reduzieren, oder wenn es gelang, durch Oxydation mittels Bromwasser zu einem Körper zu kommen der eine bromsubstituierte Methyltaurocarl aminsäure darstellte.

Die erste Formel hingegen mufste, als dem Thiosinaminbromid zukommend, anerkannt werden, wenn das Reduktionsprodukt nicht identisch mit dem $\beta$ Methyläthylen $\psi$ Thioharnstoff, sondern vielmehr mit einem wirklichen Propylen $\psi$ Thioharnstoff war.

Gelang es nicht einen derartigen Propylen $\psi$ Thioharnstoff zu erhalten, so blieb noch ubrig, umgekehrt zu versuchen, einen $\beta$ Monobrompropylen $\psi$ Thioharnstoff durch Umlagerung von Bromthiosinamin darzustellen.

Einwirkung von $H$ alogenwasserstoffaut Thiosinamin in der Kält $\theta$.

Wird Thiosinamin mit rauchender Brom- oder Chlorwasserstoffsäure bei $100^{\circ}$ in einer Druckflasche eine Stunde erhitzt, so geht dasselbe, wie $\mathrm{G}$ a b r i $\theta \mathrm{l}$ angiebt, in den halogenwasserstoffsauren $\beta$ Methyläthylen $\psi$ Thioharnstoff über. Der Vorgang verläuft nach folgendem Schema :

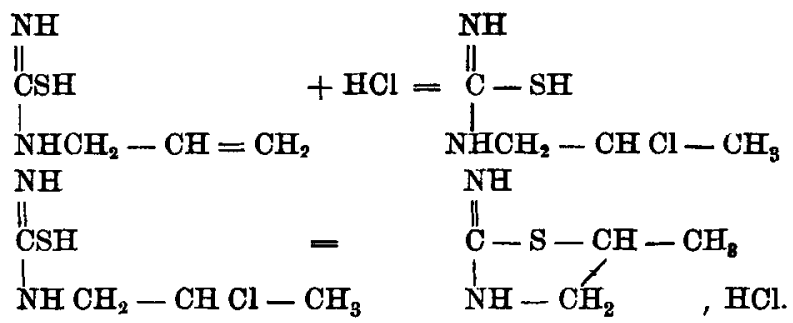

Es wird also, unter Lösung der doppelten Bindung der Allylgruppe, zunächst $\mathrm{HCl}$ addiert, und zwar Chlor in der Mitte, $\mathrm{H}$ am Ende der Allylgruppe, und die dadurch entstehende Verbindung lagert sich im Augenblick des Entstehens, unter Ringbildung, in halogenwasserstoffsauren $\beta$ Methyläthylen $\psi$ Thioharnstoff um. Nun mufs es immerhin fraglich erscheinen, ob ein gleicher Prozels bei 
Einwirkung von Halogenwasserstoff in der Kalte vor sich gehen wiirde, oder ob sich da nicht das Halogen am Ende und der Wasserstoff in der Mitte anlagern könnte, und die dabei entstehende Verbindung beim Erwarmen alsdann ebenfalls unter Ringbildung eine Umlagerung zu halogenwasserstoffsauren Propylen $\psi$ Thioharnstoff erfahren wtirde. Allerdings sind bisher fast keine Verbindungen mit doppelter Bindung bekannt, welche den Halogenwasseratoff in dieser Weise zu addieren vermöchten, vielmehr gilt als Rogel, dafs das Halogenatom immer an dasjenige Kohlenstoffatom tritt, welches mit den wenigsten Wasserstoflatomen verbunden ist.

Um dies za ermitteln, loste ich $5 \mathrm{~g}$ Thiosinamin in möglichst wenig starker Bromwasserstoffasure and leitete dann in diese Lösung, unter guter Kuhlung, so lange Bromwasserstoff ein, bis nichto mehr absorbiert wurde. Es resultierte eine sirupartige, brăunliche Flussigkeit, aus der sich aber freiwillig keine Krystalle abschieden. Ich liefs nunmehr über Aetzkalk im luftverdünnten Raumo verdunsten. Da aber, selbst nach monatelangem Stehen, keine $\mathbf{K}$ rystallisation eintrat, verdampfte ich einen Teil der sirupartigen Fltissigkeit anf dem Wasserbade, bis sich nur noch geringe Mengen von Bromwasserstoff entwickelten, verdiunnte mit Wasser, setzte Chlorsilber, zur Umwandlung des Bromids in dus Chlorid, zu und fuhrte das Filtrat in das Platinsalz tber. Auf Zasatz von Platinchlorid schied sich zunłahst nichts ans, sondern erst beim Verdunsten ther Sohwefelsanre. Ich erhielt so gat ausgebildete, grofse Krystalle von rechteckiger Form, dis wasserfrei waren, bei ca. $205^{\circ}$ ohne zu schmelzen verkohlten und von der Farbe des Kalinmdichromats paren.

0,4234 lieferten beim Glahen 0,1288 Pt.
Gef.
Pt 30,42
Ber. fur $\left[\mathrm{C}_{4} \mathrm{H}_{8} \mathrm{~N}_{8} \mathrm{~S} . \mathrm{HCl}\right]_{9} \mathrm{PtCl}_{4}$ 30,3 .

Das Platinsalz entspricht demnach vollkommen dem des $\beta$ Methylaethylen $\psi$ Thioharnstoffes $\mathrm{Ga}$ b ri $\mathrm{el}$ s.

Da nun die Moglichkeit nahe lag, dafs sich zwar znerst ein Additionsprodakt gebildet habe, dieses sich aber beim Eindampten anf dem Wasserbade in den bromwasserstoffanuren $\beta$ Methyleethylen $\psi$ Thioharnstoff umgelagert habe, so verdunnte jch das urspringliche Eirowirkungsprodukt mit Wagser und sotzte es mit Chlorsilber um. Das Filtrat verwandelte ich in das Platin-, Goldsale und das Pikrat. 
Das dabei erhaltene Platinsalz glich in allen seinen Eigenschaften dem obigen.

$0,4838 \mathrm{~g}$ des zerriebenen und bel $100{ }^{\circ}$ getrockneten Platinsalzes binterlielsen beim Glahen $0,1403 \mathrm{~g} \mathrm{Pt}$.
Gef.
Pt 30,24
Ber. fur $\left[\mathrm{O}_{4} \mathrm{H}_{8} \mathrm{~N}_{8} \mathrm{~S} . \mathrm{HCl}_{2}\right]_{2} \mathrm{PtCl}_{6}$ 30,3 .

Auf Zusatz von Goldchlorid entstand zunkchst ein amorpher, gelbroter Niederschlag, der sich beim Erwarmen klar löste und beim Erkalton in gelbroten, federigen Blattchen auskrystalliaierto. Der Schmelzpunkt lag bei $192{ }^{\circ}$. Das Salz ist wasserfrei und zersetzt sich bei $100^{\circ}$ bereits etwas.

$0,2973 \mathrm{~g}$ des uber Schwefeleture getrockneten Salzes hinterliefsen beim Gluhen $0,1274 \mathrm{~g}$.
Gef.
An 42,85
Ber. fur $\mathrm{C}_{4} \mathrm{H}_{8} \mathrm{~N}_{3} \mathrm{~S} . \mathrm{HCl}$. $\mathrm{AuCl} \mathrm{I}_{3}$ 43,14 .

Das Pikrat fiel zunischst als amorpher, kagiger Niedersoblag ans; beim Umkrystallisieren aus heifsem Wasser orhielt ich $\theta 8$ in federbartartigen Krystallen vom Schmelzpunkt $190^{\circ}$. Gabriol giebt für das Pikrat des $\beta$ Methylaethylen $\psi$ Thioharnstoffa als Schmelzpunkt: $198-200^{\circ}$ an.

Die Krystallformen der Salze, ihre Schmelzpunkte und die analytischen Daten beweisen, dafs Bromwasserstoff bereits bei gewöhnlicher Temperatur das Thiosinamin in dem Sinne Ga b ri el's umza. lagern vermag. Dieselbe Erfahrung habe ioh bei der Einwirkung von Jodwasserstoff gemacht. Das darans dargestellte Goldsalz hatte genan dieselbe Krystallform, schmolz aber bereits bei 186-1880. Der Schmelzpankt des Pikrates lag, ubereinstimmend mit dem des durch Einwirkung von Bromwasserstoff dargestallten Salzes, bei 1990.

Diese Versuche lieferten demnach keinerlei Beitrage zur Kenntnis der Konstitution des Thiosinaminbromid's. Hingegen führten dieselben zur Entdeckung eines Irrtums, in dem sich Will und Falke befunden haben. Fs handelt sich um das Platinsalz des Thiosinamins und das salzsaure Thiosinamin.

Will giebt an $\left.{ }^{1}\right)$ „Fłallt man eine mit Salzsăure versetzte Auf16sung von Thiosinamin mit Platinchlorid, so orhalt man gelbrote Niederschläge, die jedoch, je nach der Bereitungsweise, nicht immer

1) Ann. f. Chem. u. Pharm. 52, 11. 
von gleicher Zusammensetzung sind. Einen schön krystallinischen Niederschlag von konstanter Zusammensetzung erhalt man nur dann, wenn das Thiosinamin mit salzsaurem Gas gesattigt und die Auflosung mit Platinchlorid kalt gefallt wird." Auf diese Weise orhielt W ill Salze von 30.3-31\% Platingehalt. Der Umstand, dals Will die kalto Fällung mit Platinchlorid betont, lärst daranf schliefsen, dafs er den Chlorwasserstoff nicht blos in der Kälte, sondern auch in der Warme hat einwirken lassen.

Demgemäls leitete ich über trockenes Thiosinamin, das sich in einem Urohr befand, einen getrockneten Strom von Chlorwasserstoff, orgt in der Käte, dann bei 1000. Nach ca. sechsstundiger Einwirkung loste ich die gelbe, zhhe Masse in Wasser und versetzte einen Teil davon mit wässriger Pikrinsäure. Ich erhielt einen amorphen Niederschlag, der sich beim Umkrystallisieren aus heifsem Wasser in denselben federigen Krystallen ausschied, wie G a b ri e l's pikrinsaurer $\beta$ Methylaethylen $\psi$ Thioharnstoff, und deren Schmelzpunkt ebenfalls bei $199-200^{\circ}$ lag.

Einen andern Teil versetzte ich mit Platinchlorid. Es entstand sofort ein geringer, amorpher Niederschlag, den ich abfiltrierte, Im Filtrat schieden sich beim Stehen über Schwefelsüure, neben geringen Mengen einer amorphen Verbindung, gelbrote Krystalle aus, die nach dem Umkrystallisieren aus salzsäurehaltigem Wasser in derselben, nur etwas weniger scharf ausgepragten Krystallform und von der gleichen Farbe resultierten, wie das $\beta$ Methylaethylen $\psi$ Thioharnstoff-Platinchlorid.

$$
\begin{aligned}
& 0,1963 \mathrm{~g} \text { hinterliefsen beim Gluhen } 0,0601 \mathrm{~g} \mathrm{Pt} \text {. } \\
& \text { Pt Gef. } \quad \text { Ber. fur }\left(\mathrm{C}_{4} \mathrm{H}_{8} \mathrm{~N}_{\mathrm{g}} \mathbf{8 .} \mathrm{H} \mathrm{Cl}\right)_{2} \mathrm{Pt} \mathrm{Cl}_{4}
\end{aligned}
$$

Daraus geht anzweifelhaft hervor, dafs das konstant zusammengesetate Thiosinaminplatinchlorid Will's nichts anderes als salssaures $\beta$ Methylaethylen $\psi$ Thioharnstoff-Platinchlorid ist. Ebenso ist das von F a l ke nach Will's Vorschrift dargestellte salzsaure Thiosinamin das Chlorhydrat obiger Base, welches bei einem geringen Fenchtigkeitsgehalt uberschtssigen Chlorwasserstoff mechanisch festhät. Dadurch erklart sich die eigentümliche Verbindung von einem Molekil Thiosinamin mit nicht ganz zwei Molektalen Salzsaure.

Bei dieser Gelegenheit möchte ich erwhhnen, dafs ich ziemlich konstant zusammengesetzte Platindoppelsalze des Thiosinaming er- 
halten habe, wenn ich eine wässrige Lösung des Thiosinamins (1:150) mit therschlissigem Platinchlorid versetzte. Diese Platinsalze waren fein-krystallinisch, von orangegelber Farbe und zersetzten sich bereits beim Trocknen im Wassertrockenschrank.

Daten :

Zwei verschiedene Praparate gaben bei der Analyse folgende

1. 0,1979 g des Uber Schwefels ăure getrockneten Praparateo hinterliefsen $0.0720 \mathrm{~g} \mathrm{Pt}$.

2. 0,2426 derselben Substanz gaben $0,1925 \mathrm{~g} \mathrm{Ag} \mathrm{Cl}$.

3. 0,2968 $\mathrm{g}$ eines anderen, ebenfalls uber Schwetelshure getrockneten Präparates hinterliefsen beim Glaten $0,1086 \mathrm{~g}$ Platin.

\begin{tabular}{cccc}
\multicolumn{3}{c}{ Gefunden: } & Berechnet fur \\
I & II & III & {$\left[\mathrm{C}_{4} \mathrm{H}_{8} \mathrm{~N}_{8} \mathrm{~S}_{2} \mathrm{Pt} \mathrm{Cl}_{2}+\mathrm{HCl}\right.$.} \\
Pt 36,38 & - & 36,6 & 36,5 \\
Cl - & 19,63 & - & 20,0
\end{tabular}

Ob aber obige Formel dem Thiosinaminplatinchlorid wirklich zukommt, möchte ich dahingestellt sein lassen, da die Zusammensetzung des Salzes allzusebr von den Verhaltnissen abhăngig ist, unter denen es dargestellt wird.

Ein Salz ron zhnlicher Zusammensetzung erhielt ich beim Fallen einer wässrigen Thiosinaminlösung, die mit dem gleichen Volumen 25 prozentiger Salzsăure versetzt war. 35,88 Prez.

0,2533 g dieses Praparates gaben 0,0909 g Platin, entsprechend

Bei einer späteren Gelegenheit werde ich noch einmal auf diese Verbindungen zurïckkommen.

Darstellung von Bromgenfölund Bromthiosinamin.

$250 \mathrm{~g}$ Tribrompropan vom Siedepunkt $200^{\circ}$ vermischte ich mit $500 \mathrm{~g}$ wasser- und alkoholfreien Aethers, dargestellt durch Ausschuitteln des gewöhnlichen, kăuflichen Aethers mit Wasser, Entwässern mit Chlorcalcium und Destillation tuber metallisches Natrium, und figte dazu, nach Angabe von Tollens ${ }^{1}$ ), etwas mehr, als die zur Ueberführung des Tribrompropan in Dibrompropylen ertorderliche Menge metallisches Natrium in dünnen Scheiben hinzu. Berechnet waren $20,5 \mathrm{~g}$ Natrium. Ich konnte dabei nicht, wie Tollons,

1) Annal. für Chem. u. Pharm. 156, 168. 
eine Entwickelung selbstentzündlicher Gase konstatieren, vielmehr war die Einwirkung eine sehr allmăliche, bei geringer Warme- and Waserstoffentwickelung. Die ron Tollens bemerkten Feuererscheinungen können daher nur, wie er selbst vermutet, von dem Vorhandensein gröfserer Quantitäten Wasser herruhren. Uebereinstimmend mit Tolleng beobachtete ich indessen, dals aich das Natrium mit einer intensiv indigoblauen Schicht uberzog, welche bei dem weiteren Verlanf der Reaktion gelblich weifs warde. Nach mehrtagiger Einwirkung in der Kälte, erhitzte ich dann das Gemisch am Rtickflufsktuhler auf dem Damptbade so lange, bis alles metallisches Natrium in Bromnatrium ubergefubrt war. Die Flüsigkeit wurde von letzterem abfiltriert und mit Aether nachgewaschen. Dabeimachtesich ein eigentiumlich stechender Geruch bemerkbar, welcher auch die Augen sehr angriff and dieselben zu Thranen reizte. Der Aether warde im Wasserbade abdestilliert (derselbe enthielt betrichtliche Mengen des stechend riechenden Körpers), und der Rtickstand in einem Siedekolben der fraktionierten Destillation unterworfen. Die bei 140 bis $150^{\circ}$ ubergehenden Anteile wurden gesammelt und nochmals rectifiziert, wobei die bei $140-145^{\circ}$ siedenden Anteile gesondert anfgefangen wurden. Die Destillationsrtickstände von beiden Fraktionierungen, die unverk̈ndertes Tribrompropan enthielten, wurden noch einmal in der oben angegebenen Weise mit Natrium behandelt und weiter verarbeitet. Bei der Destillation des nach der Gleichung

$$
\mathrm{C}_{3} \mathrm{H}_{6} \mathrm{Br}_{3}+\mathrm{Na}=\mathrm{C}_{8} \mathrm{H}_{4} \mathrm{Br}_{2}+\mathrm{Na} \mathrm{Br}+\mathrm{H}
$$

gebildeten Dibrompropylon ( $\alpha$ Epidibrombydrin) bemerkte ich, dafs dasselbe eine nicht anbeträchtliche Zersetzung erlitt; denn es entwickelten sich reichliche Mengen von gasförmigem Bromwasserstoff; auch fand ein fortwahrendes Steigen und Fallen der Temperatur statt. Dafs aber das resultierende Produkt in der Haupteache aus dem gewtinschton a Epidibromhydrin bestand, konstatierte ich durch eine Bestimmung des Bromgehalts.

$0,5816 \mathrm{~g}$, mit Salpetersllurehydrat und Silbernitrat 3 Stunden auf 170-1800 orhitzt, lieferten $1,048 \mathrm{~g} \mathrm{Ag}$ Br.
Br Gefunden: 78,49
Berechnet fir $\mathrm{C}_{8} \mathrm{H}_{4} \mathrm{Br}_{2}$ 80,00 .

Die Ausbente betrug wenig mehr als $80 \mathrm{gr}$. 
Ueberfahrung deg a Epidibrombydring in Bromsenfol.

Zur Ueberfuhrung des $\alpha$ Epidibrombydrins in Bromsenföl verfuhr ich nach den Argaben von $\mathrm{H}$ o $\mathrm{n} \mathrm{r}^{\mathbf{2}}$ ).

a Epidibromhydrin wurde mit der füffachen Menge Alkohol verdünnt und, nach Zusatz einiger Gramm Kaliumacetat (zur Bindung freier Bromwasserstoffsulure), mit der berechneten Menge Rhodankalium versetzt. Schon in der Kulte fand eine reichliche Abscheidung von Bromkalium statt. Zur Beendigung der Reaktion wurde das Gemisch noch einige Stunden am Ruckflufskuhler gekocht, wobei die Flussigkeit eine dunkelbraune Furbung annahm, welche bereits bei der Einwirkng in der Kalte in geringem Malse aufgetreten war. Nach dem Abfiltrieren vom ausgeschiedenen Bromkalium destillierte ich den Alkohol möglichst ab und jagte das gebildete Bromsenföl mit Wasserdămpfen uber. Als das whissrige Destillat nicht mebr milchig, sondern klar abtropfte, vereinigte ich dasselbe und entzog ihm das Bromsenföl mit Aether. Nach dem Verdunsten desselben verblieb ein schwach bräanlich gefarbtes Liquidum, das einen intensiven Senfölgeruch besafs. Der Versuch, einen Teil desselben durch direkte Destillation zu rektifizieren, mifslang, da schon bei etwa $170^{\circ}$ eine tietgreifende Zersetzung, unter Abscheidung von Koble, stattfand. Diese Zersetzung mufs ihren Grund in beigemengten Verunreinigungen haben, da dem reinen Bromsenfol, nach Henry, der Siedepunkt $200^{\circ}$ zukommt. Da es mir jedoch nur auf das Bromthiosinamin ankam, so verzichtete ich auf eine weitere Reinigung des Senfols, und ich versuchte, das Rohprodukt durch Behandeln mit alkoholischem Ammoniak in ersteres überzuführen.

Ueberfühung des Bromenföls in das Brom thiosinamin.

Das Bromsenföl wurde mit uberschussigem, alkoholischen Ammoniak von 10 Proz. versetzt. Es entstand dadurch eine klare, schwach gefärbte Lösung; eine Einwirkung des Ammoniaks auf das Senfol konnte jedoch nicht bemerkt werden; auch gelinde Erwärmung, welche doch genügt, um gewöhnliches Allylsenföl in Thiosinamin zu verwandeln, führte hierbei nicht zum Ziele, vielmehr mufste ich die

1) Ber. V, 188. 
Mischnng stundenlang auf dem Damptbade erhitzen and das dabei verdunstete Ammoniak mehrmals ersetzen.

Als das Cremisch dann nicht mehr nach Senföl roch, wurde es auf ein kleines Volumen eingeengt. Beim Erkalten schieden sich reichliche Mengen von Krystallen aus, die in eine schwarzbraune zkhe Masse eingebettet waren und von letzterer durch Abpressen zwischen Thonplatten getrennt wurden. Durch Umkrystallisieren aus verdünntem Alkohol erhielt ich weifse, zu sternförmigen Drusen vereinigte Krystallnadeln. Ihr Schmelzpunkt lag bei $111^{0}$, stimmte also mit dem von $\mathrm{H}$ en ry für das Bromthiosinamin angegebenen uberein. Zur weiteren Identifizierung bestimmte ich den Brom- und Schwefelgehalt nach $\mathrm{Car}$ i us.

$0,3971 \mathrm{~g}$ gaben dabei $0,3800 \mathrm{~g} \mathrm{Ag} \mathrm{Br}$ und $0,4738 \mathrm{~g} \mathrm{Ba} \mathrm{SO}$. Gefunden :

Br 40,72 Berechnet fur $\mathrm{C}_{4} \mathrm{H}_{7} \mathrm{Br} \mathrm{N}_{8} \mathrm{~S}$

S $\quad 16,39$

16,41 .

Versuch, das Bromthiosinamin in don isomeron $\psi$-Thioharnstoff umzulagern.

Nachdem ich so unzweifelhaft die Identität des erhaltenen Produktes mit Henry's Bromthiosinamin bewiesen hatte, versuchte ich dasselbe nach der von Gabriel angegebenen Methode in den isomeren Pseudothioharnstoff umzulagern.

Dem Bromthiosinamin kommt nach der vorbeschriebenen Darstellungsweise die Konstitutionsformel

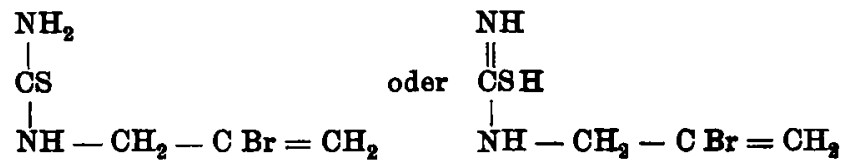

zn, wenn wir auch hier wieder zwei tautomere Formen annehmen. Bei Annahme der letzteren, die, mit Rücksicht auf das Thiosinamin, entschieden den Vorzug verdienen mufs, wird zu erwarten sein, dafs das Bromthiosinamin beim Erhitzen mit ranchender Chlor- oder Bromwasgerstoffsäure zunächst durch Aufhebung der doppelten Bindung in einen Körper von der Konstitution

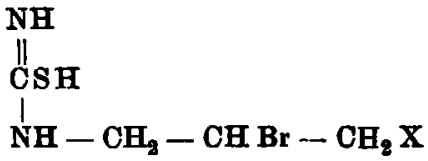


übergehen wird, der dann analog Gabriel's $\beta$-Methyläthylen$\psi$-Thioharnstoff eine Umlagerung in den halogenwasserstoffsauren Monobrompropylen- $\psi$-Thioharnstoff erfahren wird. Die dabei entstehenden Körper könnten dann, je nach Anwendung des Chloroder Bromwasserstoffs mit dem M al y'schen Thiosinaminbromochlorid oder Thiosinaminbromid identisch sein.

Ich erhitzte also $2 \mathrm{~g}$ Bromthiosinamin mit $5 \mathrm{ccm}$ rauchender Salzsäure $1 \frac{1}{2}$ Stunde lang in einer Druckflasche auf $100^{\circ}$. Darauf vermischte ich die erkaltete, klare Flüssigkeit, in der sich alles Bromthiosinamin gelöst hatte, mit Wasser. Es entstand eine milchige Trübung, welche beim Erwärmen verschwand. Beim Erkalten schieden sich wieder weifse Krystallmassen aus, deren Schmelzpunkt bei $111^{\circ} \mathrm{lag}$, die also aus unverändertem Bromthiosinamin bestanden. Die Mutterlaugen liels ich über Aetzkalk verdunsten; es blieb schlielslich ein gelblicher Sirup zurück, der mit wenig Wasser sich klar mischte, auf Zusatz von viel Wasser aber ebenfalls weifse Krystalle ausschied, die, wie der Schmelzpunkt lehrte, aus unverändertem Bromthiosinamin bestanden. Kine Addition von $\mathrm{HCl}$ und eine Umlagerung im Sinne der Gabriel'schen Gleichung hatte also nicht stattgefunden.

Einen anderen Teil schloss ich mit rauchender Salzsäure in ein starkwandiges Glasrohr ein und erhitzte ihn zwei Stunden lang auf $120-130^{\circ}$. Das Produkt nahm ich mit Wasser auf, worin es sich, abweichend von dem nur auf $100^{\circ}$ erhitzten Gemisch, völlig klar auflöste. Auf dem Dampfbade eingedampft, verblieb ein gelblich brauner Sirup, der selbst nach wochenlangem Stehen über Schwefelsäure nicht krystallinisch exstarrte, auch nicht, als ich in den Sirup eine geringe Menge des nach $\mathrm{M}$ a 1 y dargestellten Thiosinaminbromochlorids eintrug. Beim Stehen an feuchter Luft wurde der Sirup wieder dünnflüssig, während Thiosinaminbromochlorid unter gleichen Bedingungen unverändert blieb. Somit konnte das gebildete Produkt nicht mit letzterem identisch sein; vielmehr ist anzunehmen, dals die rauchende Salzsäure bei $120-130^{\circ}$ tiefer zersetzend auf das Bromthiosinamin eingewirkt habe, wie ich mit Sicherbeit daraus entnehmen kann, dals das Einwirkungsprodukt auf Zusatz von Natronlauge einen intensiven Geruch nach Ammoniak annahm. 
Beim Behandeln von Thiosinamin mit gastörmigem Bromwasserstoff, und ebenso mit Jodwasserstoff, hatte ich konstatiert, dafs diese beiden Säuren bereits in der Kälte eine Umlagerung zu halogenwasserstoffsaurem $\beta$ Methylaethylen $q$ thioharnstoff bewirken. Da ferner das Thiosinaminbromid sich durch grölsere Krystallisationsfähigkeit auszeichnet, als das Bromochlorid, so versuchte ich endlich, Bromthiosinamin in analoger Weise mit $\mathrm{H} \mathrm{Br}$ umzulagern.

$\mathrm{Zu}$ diesem Behufe schüttelte ich $2 \mathrm{~g}$ Bromthiosinamin mit $5 \mathrm{ccm} 25$ prozentiger Bromwasserstoffsäure in einer starkwandigen Flasche an und leitete dann so lange gasförmigen Bromwasserstoff ein, bis nichts mehr absorbiert wurde; dabei löste sich das Bromthiosinamin völlig auf. Darauf erhitzte ich die Lösung in der verschlossenen Druckflasche ca. 2 Stunden auf $100^{\circ}$. Das Finwirkungsprodukt war gelblich gefärbt. Einen Teil desselben dampfte ich auf ein kleines Tolumen ein und verdünnte mit Wasser. Dabei schieden sich bräunlich gefärbte, harzige Massen ab, die nach einiger Zeit zum Teil krystallinisch erstarrten. Der gröfste Teil blieb in Lösung. Beim Verdunsten über Schwefelsäure schieden sich stark glänzende, undurchsichtige, weifse Krystallschuppen aus, dif bei 213-2150 unter Gasentwickelung schmolzen. Mithin war eine Veränderung des ursprünglichen Körpers vor sich gegangen; doch konnte die erbaltene Verbindung nicht mit $\mathrm{M}$ a l y's Thiosinaminbromid identisch sein, da dieses bereits bei $139^{\circ}$ schmilzt.

Das Material reichte leider nur zu einer Bestimmung des Brom- und Schwefelgehalts nach Carius zu.

$0,2038 \mathrm{~g}$ lieferten dabei $0.2048 \mathrm{~g} \mathrm{Ag} \mathrm{Br}$ und $0,2542 \mathrm{~g} \mathrm{Ba} \mathrm{SO} 4$.
Gefunden
$\mathrm{Br} 42,76$
$\mathrm{~S} 1 \overline{1}, 13$

Das unveränderte Bromthiosinamin erfordert $41,02 \mathrm{Br}$ und 16,41 Proz. S. Diese Differenzen, sowie der bedeutend höher liegende Schmelzpunkt weisen auf eine Teränderung der Substanz hin; dieselbe konnte jedoch nicht im Sinne Gabriel's ertolgt sein, da eine solche Verbindung 57,97 Proz. Brom und 11,59 Proz. Schwefel voraussetzt. Nun enthielten die Mutterlaugen von obigem Körper reichliche Mengen von Ammoniumbromid neben geringen Mengen von Aminsalzen, wie ich beim Uebergielsen derselben mit 
Natronlange deatlich am Geruch erkennen konnte. Um dieselben aber noch genauer nachzuweisen, destillierte ich die Mutterlaugen mit Natronlauge und fing das Destillat in verdunnter Salzsăure auf. Das Destillat wurde anf ein kleines Volnmen eingedampft und mit Platinchlorid versetzt. Nach einiger Zeit schieden sich octoedrische Krystalle aus, die in ibrer Form vollstandig dem Platinsalmiak glichen. Von dem getrockneten und zerriebenen Präparat versuchte ich den Schmelzpunkt zu bestimmen. Bei 2600 jedoch war die Substanz weder geschmolzen, noch zeigte sie sonst bedeutende Zersetzung. Da nun die Platinsalze der in Frage kommenden Aminbasen sämtlich bei niedrigerer Temperatur schmelzen, so mufsten die Krystalle aus Platinsalmiak bestehen. Somit war erwiesen, dafs konzentrierte Bromwasserstoffedure auf Bromthiosinamin ammoniakentziehend einwirkt, und zwar, wie die obigen analytischen Daten ergeben, entzieht dieselbe anscheinend aus zwei Molekülen Bromthiosinamin ein Molekül Ammoniak, so dafs dadurch ein Körper entsteht, der sich zum Bromthiosinamin vielleicht verhalt, wio das Biuret zum Harnstoff. Eine derartige Verbindung würde 42,89 Proz. Brom und 17,16 Proz. Schwefel verlangen.

Diese Thatsachen veranlassen mich za der Annahme, dals dem Bromthiosinamin nicht die unsymmetrische Formel mit der Gruppe C-S.H, sondern die symmetrische mit der Gruppe $\mathrm{C}=\mathrm{S}$ zukommt.

Verhalten desthiosinaminjodids resp. -bromids gegen Reduktionsmittel.

1. Gegen $Z$ inn und $S a l z s a ̆ r e$.

Der Versuch, das Jodid mit Zinnfolie und Salzsăure zu reduzieren, führte zu keinem Resultat, da infolge der dabei notwendigen Erwarmung eine tiefer greifende Zersetzung eintrat.

2. Gegen Natrium-Amalgam.

Durch Reduktion des Thiosinaminbromids mit Natriumamalgam in wassriger Lobsung hat Falke ein Prlparat erhalten, dessen Platinsalz in seiner prozentischen Zusammensetzung annahernd mit dem des $\beta$ Methylaethylen $\psi$ Thioharnstoffs vibereinstimmte. Die physikalischen Eigenschaften waren aber so wesentlich abweichend,

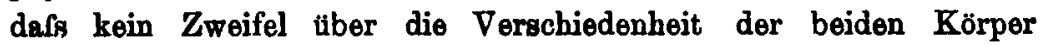


herrschen konnte. F a lke verfuhr bei der Roduktion in der Weise, dals or das Natriumamalgam in kleinen Portionen in die wassrige Lösung eintrug, so dafs eine rubige Wasserstoffentwickelung stattfand. Bei allzu heftiger Einwirkung bemerkte er einen Geruch nach Cyan und Knoblauch, sowie die Abscheidung schmutzig graner, zkher Massen. Erst nach beendigter Reduktion neutralisierte or mit Salzsturure.

Ich modifizierte sein Verfahren, von der Annahme geleitet, dafs die stark alkalische Flüssigkeit eine wesentliche Verunderung des Reduktionsproduktes herbeigeführt haben könnte, in der Art, dals ich, sobald die Einwirkung eines kleinen Stúckchens Natriumamalgam beendet war, erst mit Salzsăure sorgtăltig neutralisierte, bevor ich neue Mengen von Natriumamalgam zusetzte. Dadurch vermied ich eine Zersetzung, wie sie sich durch den Geruch nach Cyan und Knoblauch bemerkbar gemacht hatte; bingegen fand auch hier eine geringeAbscheidung grülich grauer, zaher.Massen statt. Nach etwa achttagiger Einwirkung filtrierte ich die angestuerte Fltissigkeit ab, versetzte sie mit Chlorsilber, dampfte, zur möglichsten Abscheidung des Chlornatriums, zur Trockne ein und extrahierte dann mit absolutem Alkohol. Das alkoholische Filtrat befreite ich vom Alkohol durch Erwärmen, nahm den Rückstand mit viel Wasser auf and verwandelte einen Teil desselben in das Platinsalz, einen andern in das Goldsalz.

Das Platinsalz fiel amorph aus; und zwar konnte man zwei verschieden getärbte Niederschläge deutlich unterscheiden, eine hellgelbe Fallung, die sich zuerst abschied, und daruber eine tieforangegelbe. Eine Trennung der beiden Niederschläge durch Umkrystallisieren gelang nicht. Die davon ausgeführten Analysen bestätigen die Annahme, dofs man es mit einem Gemisch verschiedener Salze zu thun hat. Eine Beimengung von Halogennatrium konnte ich jedoch nicht konstatieren.

1. $0,1743 \mathrm{~g}$ Substanz lieferten $0,0520 \mathrm{~g} \mathrm{H}_{2} \mathrm{O}$ und $0,0790 \mathrm{~g} \mathrm{CO}$.

2. $0,2293 \mathrm{~g}$ von einer andern Fällung stammend, lieferten $0,0545 \mathrm{~g} \mathrm{H}_{8} \mathrm{O}$ und $0,1140 \mathrm{~g} \mathrm{CO}_{8}$.

3. $0.2035 \mathrm{~g}$ gaben $0,2177 \mathrm{~g} \mathrm{Ag} \mathrm{Cl}$ und $0,0713 \mathrm{~g}$ Platin.

4. $0,1776 \mathrm{~g}$ hinterliefsen beim Gluhen $0,0647 \mathrm{~g} \mathrm{Pt}$.

5. $0,1035 \mathrm{~g}$ lieferten $0,1105 \mathrm{~g}$ Ag Cl. 


\begin{tabular}{|c|c|c|c|c|c|c|}
\hline & \multicolumn{3}{|c|}{ Gefunden: } & \multicolumn{3}{|c|}{ Quotienten: } \\
\hline & I & II & III & IV & $\nabla$ & \\
\hline C & 12,36 & 13,56 & - & - & - & $5-6$ \\
\hline 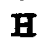 & 3,31 & 2,64 & - & - & - & -16 \\
\hline $\mathrm{G}$ & $\rightarrow$ & - & 26,46 & - & 26,41 & 4 \\
\hline D & - & - & 35,04 & 36,43 & - & 1. \\
\hline
\end{tabular}

Die fur Chlor und Platin gefundenen Werte sprechen für ein Platinosalz, welches etwa in ahnlicher Weise zusammengesetzt ist, wie Falke's Thiosinaminplatinchlorid. Die Farbe des Salzes entsprach demselben vollkommen. Die fur Kohlenstoff gefundenen Werte weichen aber so wesentlich ab, dafs von einer einheitlichen Verbindung nicht die Rede sein kann. Die Verbrennungen wurden direkt im Sanerstoffstrome vorgenommen.

Das Goldsalz fiel als ein branes, amorphes Pulver aus, verkohlte ohne zu schmelzen und liefs sich weder aus salzsäurehaltigem Wasser, noch aus Alkohol umkrystallisieren.

$0,5236 \mathrm{~g}$ hinterliefsen beim Gltahen $0,3442 \mathrm{~g}$ Gold. Dies ent. opricht einem Gehalt von 65,74 Proz.

Daraus geht hervor, dafs der aus dem Thiosinaminjodid durch Reduktion dargestellte Körper stark reduzierende Eigenschaften bositzen mufs, keinesfalls aber identisch mit dem $\beta$ Methylkthylen $\psi$ thioharnstoff, oder demselben anch nur ahnlich sein kann.

3. Gegen metallisches Natriam in alkoholischer L $8 \mathbf{s} \mathbf{n g}$.

Za einer alkoholischen Lösung des Thiosinaminjodids flugte ich nnter guter Abktihlung allmählich etwa die vierfache Menge metallisches Natrinm hinzu.

Ein Geruch nach Cyan und Knoblauch, wio ihn Falke bei Roduktion de Thiosinaminbromids mit Natrinmamalgam in whssriger Lobsung wahrnahm, trat dabei nicht auf.

Als die Einwirkung in der Kalte nur noch eine sehr trage war, erwämte ich gelinde die Fltussigkeit, die allmahlich zu einem steifen Brei erstarrte. Auf Zusatz von Wasser loste sich derselbe vollKommen klar auf. Die klare Lősung wurde mit Salzołure neutralisiert, mit Chlorsilber umgesetzt und nach dem Filtrieren eingedampft. Dabei schieden sich am Rande klebrige, gelbgefärbte, harzartige Massen aus, die ich zunlichst unbeachtet liefs. Die chlornatriom- 
baltige Lösung wurde zur Trockne eingedampft und mit Alkohol extrahiert. Das alkoholische Extrakt wurde mit Wasser verdunnt und zur Verjagang des Alkohols eingedampft. Einen Teil der noch etwas Chlornatrium enthaltenden klaren Losung versetzto ich mit Platinchlorid. Es fiel dadurch ein amorpher, gelber Niedersohlag aus. Einen andern Theil versetzte ich, um ihn vollig frei von Chlornatrinm zn bekommen, mit Wismutjodidjodkalium. Den dabei entstehenden Niederschlag saugte ich ab, verrieb ihn mit feuchtem Barynmkarbonat und filtrierte, nach dem Verdunnen mit Wasser, die Lobsung klar ab.

Das darin als Chlorid resp. Jodid enthaltene Baryum entfernto ich durch einige Tropfen Schwefelsanre und die dadurch frei werdende Jodwasserstoffsinure durch Umsetzen mit Chlorsilber. Die dadurch erhaltene salzsaure Lösung der Base fallte ich endlich mit Platinchlorid. Der Niederschlag war ebenfalls amorph und glich dem obigen, direkt erhaltenen vollatändig. Die Ausbeute war aber in beiden Fællen eine so geringe, dafs eine Analyse nicht möglich war, und stand in keinem Verhaltmis zum angeviandten Thiosinaminjodid.

Ich schritt nun zur Untersuchung der oben erwshnten, braunlichen, harzigen Massen, die sich aus der chlornatriumbaltigen Fltissigkeit abgeschieden hatten. Nach Entfernung der Salzlösung und Nachwaschen mit otwas Wasser, erwies sich das harrige Produkt als loblich in Wasser und verdtinnter Salzsture. Die wässerige Lösung wurde jedoch wieder als weilser, flockiger Niederschlag durch Zusatz von Kochsalzløsung gefullt. Eine salzsaure Lösung liefs ich treiwillig auf einem flachen Uhrglase verdunsten; doch erhielt ich dedurch keine Krystalle, vielmehr trooknote die Fltissigkeit nur 2u einer durchscheinenden, amorphen, braunen Masse ein.

Ich fuhrte deshalb die salzsaure Losung in das Gold- und Platingals tiber.

Das Goldsalz bildete ein brannes, fein krystallinisches Pulver. Beim Trocknen ther Sohwefelsture verlor fast nichts an Gewicht, hingegen gab es bei $100^{\circ}$ bedeutend ab, whhrend sich gleichreitig eine dankjere Furbung einstellte, so dafs eine Zersetzang des Goldsalzes anzunehmen war. 
1. $0,2868 \mathrm{~g}$ verloren uber Sohwefelshure $0,0043 \mathrm{~g}$. durch Trocknen bei $100^{\circ} 0,0275 \&$ oder 9,59 Proz. Dieselbe Menge hinterliefe beim Glthen 0,1398 \& Gold.

2. $0,2069 \mathrm{~g}$ der getrockneten Substanz gaben $0,1520 \mathrm{~g} \mathrm{Ag} \mathrm{Cl}$. Gefunden :

Berechnet for

I. II.

Au 51,30

$\mathrm{C}_{4} \mathrm{~B}_{8} \mathrm{~N}_{\mathbf{8}} \mathrm{S} \cdot \mathrm{HCl} \cdot \mathrm{Au} \mathrm{Cl}$

$\mathrm{Cl}-\quad 18,22$

51,26

Demnach hatte ich es mit dem $A$ a r 08 a I z einer Base zu thun, welche mit $\operatorname{dem} G$ abriel'schen $\beta$-Methylsthylen- $\psi$-Thioharnstoff isomer war, die Analysen des Platinsalzes ftuhrten aber zu vollig anderen Resultaten. Die dabei gefundenen Werte ftlhren za der Annahme, dafs Natrium anf die alkoholische Losung des Thiosinaminjodids in der Weise eingewirkt habe, dafs sich zankachst Natriumsthylat gebildet, und dieses, unter Abscheidung von Jodnatrium, in das Thiosinaminjodid ftir Jod eine Ozathylgruppe eingefuhrt habe. Wodurch die damit nicht tibereinstimmende Zusammensetzung des Goldsalzes herbeigefthrt worden ist, vermag ich allerdings nicht zu entscheiden.

1. $0,3552 \mathrm{~g}$ des Infttrockenen, amorphen Platinualzes verloren bei $100^{0} 0,0177 \mathrm{~g} \mathrm{H}_{2} \mathrm{O}$, welches ich aber als hygroskopische Feuchtigkeit ansprechen mochte. Bis zum konstanten Gewicht geglaht, verblieben 0,0886 g Platin.

2. 0,2490 des bei $100^{\circ}$ getrockneten Salzes gaben $0,0669 \mathrm{~g}$ Platin.

3. $0.2096 \mathrm{~g}$ desselben Salzes gaben $0,1490 \mathrm{~g} \mathrm{CO}$ and 0,0698 g $\mathrm{H}_{2} \mathrm{O}$.

4. 0,1448 g derselben Subatanz gaben 0,1699 g Ag Cl.

Gefunden

Berechnet fur

\begin{tabular}{|c|c|c|c|c|c|}
\hline & 1 & II & III & IV & {$\left[\mathrm{C}_{6} \mathrm{H}_{2} \mathrm{OC}_{2} \mathrm{H}_{8}-\mathrm{N}_{2} \mathrm{~S} . \mathrm{HCl}\right]_{2} \mathrm{Pt} \mathrm{Cl}_{4}$} \\
\hline c & - & - & 19,39 & - & 18,74 \\
\hline H & - & - & 3,7 & - & 3.57 \\
\hline $\mathrm{Cl}$ & - & - & - & 29,03 & 29,20 . \\
\hline
\end{tabular}

4. Gegen Zink and Es igs a ro.

Eine alkoholische Lossung von $5 \mathrm{~g}$ Thiosinaminjodid versetste ich mit Zinkstaub and Eisessig and erwarmte das Gemisch gelinde, so dafs eine rahige, gleichmarsige Wasserstoffentwicklng stattfand. Der verdunstete Alkohol warde von Zeit zu Zeit ersetzt. Nach mehrtagiger Einwirkung warde das ungeloste Zink von der traben, rötlich gefarbten, nach Eesignther riechenden Fldssigkeit abfiltriert, and in dieselbe Schwofelwasserstoff eingeleitet, am das als Zink- 
acetat vorhandene Zink zu entfernen. Das Filtrat davon wurde durch Erwarmen von Schwefelwagserstoft betreit und darauf mit Chlorsilber behandelt. Dabei ballte sich letzteres zusammen, wkhrend gleichzeitig eine allmahliche Umsetzung za Jodsilber stattfand. Die filtrierte, jodfreie Flassigkeit war farblos und roch nach Essigsănre; anch enthielt sie natürlich etwas Zink in der Form des Chlorids, welches durch Schwefelwasserstoff nicht zu entfernen gewesen war. Finen kleinen Teil versetzte ich mit Pikrinsäure, wodurch ein amorpher, beim Erwärmen löslicher Niederschlag entstand. Beim Erkalten schied sich das Pikrat in gelbroten nicht krystallisierten Tropfchen ab. Den Rest versetzte ich mit Goldchlorid und Salzsaure. Es entstand zunächst kein Niederschlag. Beim Stehen uber Schwefelsăure schied sich lüngere Zeit metallisches Gold ab, von dem die Flüsigkeit durch Filtrieren wiederholt getrennt wurde. Endlich resultierten kugelige, rotbraune Krystalloide, die perlenschnurartig an einander gereiht waren und bei 174-1750 schmolzen. Die Ausbeute stand jedoch anch hier in keinem Verhaltnis zum angewandten 'Thiosinaminjodid, indem im ganzen nicht mehr als etwa 0,3 g des Goldsalzes gewonnen wurden. Die empirische Zusammenseteung stimmte mit der des Gabriel'schen Körpers tuberein, das Goldsalz unterschied sich aber wesentlich durch den Schmelzpunkt und die Krystallform.

0,2378 Subatanz gaben 0,3017 g Chlorsilber. Das abgeschiedene metallieche Gold wurde auf einem Filter von bekanntem Aschengehalt gessmmelt und gegluht; es betrug $0,1018 \mathrm{~g}$.

$\begin{array}{lcc}\text { Gefunden } & \text { Berechnet fur } \mathrm{C}_{4} \mathrm{H}_{8} \mathrm{~N}_{2} \mathrm{~S} \text { H Cl } . \mathrm{Au} \mathrm{Cl}_{8} \\ \mathrm{Au} & 42,81 & 43,13 \\ \text { Cl } 31,25 & 31,17\end{array}$

Da die Ausbeute an reduziertem Thiosinaminjodid eine so geringe war, so mulste dasselbe mit einem andern Körper eine unlögliche Verbindung gebildet haben, so dafs es schliefslich in den Filtraten nur noch in geringer Menge vorhanden war. Um dies zu ermitteln, reduzierte ich neue Mengen des Thiosinaminjodids in derselben Weise und beobachtete sorgfaltig alle dabei zn Tage tretenden Erscheinungen.

Ich konstatierte dabei, dafs sich an den Wandungen des Gefurses gelbe, in Wasser unlbsliche, in Alkohol nahezu unlösliche Massen absetzten. Woraus dieselben bestanden, konnto ich nicht 
ermitteln; möglich jedoch, dafs es eine Doppelverbindung von Thiosinaminjodid mit Jodzink oder von reduziertem Thiosinaminjodid mit Jodzink vorstellte. Nach ca. achttägiger Einwirknng des naszierenden Wasserstoffs wurde die Flüssigkeit vom ausgeschiedenen Zinkacetat abfiltriert und direkt, nach dem Verdünnen mit Wasser, mit Chlorsilber versetzt. "Selbiges ballte sich wiederum zu zahhen Klumpen zusammen. Beim Kochen verlor es diese zähe Beschaffenheit und wandelte sich in gelbes Jodsilber nun. Die überstehende Flüssigkeit wurde, noch heils, abfiltriert. Bein Erkalten schieden sich dann glänzend weifse, sehr voluminöse Krystallmassen aus, die aus aufserst feinen, langen Nadeln bestanden. Dieselben wurden von den Mutterlaugen abfiltriert und über Schwefelsäure getrocknet. Durch Auskochen des Rückstandes mit den Mutterlaugen konnton noch mehrfach Krystallisationen derselben Verbindung erhalten werden.

Das getrocknete Präparat war von weisser, seidengläuzender Beschaffenheit, und nur an den Stellen, wo es mit Papier längere Zeit in Berührung gewesen, war es etwas grau gefärbt. Der Schmelzpunkt lag bei $87-88^{\circ}$. Auf dem Platinblech verbrannte es unter Zurücklassung von metallischem Silber. Ich hatte es also mit einer Silberverbindung zu thun, und zwar, wie sich herausstellte, mit einer Chlorsilberverbindung.

$0,2175 \mathrm{~g}$ des zerriebenen Präparates wurden mit Salpetersllure von ca. 60 Proz. bis zur vollständigen Absoheidung des Chlorsilbers erwärmt. Die Menge desselben betrug $0,1189 \mathrm{~g}$. Gefunden: 54,67
Berecinnet fur $\mathrm{C}_{4} \mathrm{H}_{8} \mathrm{~N}_{2} \mathrm{~S} . \mathrm{Ag} \mathrm{Cl}$ 55,30 .

Im Filtrat war kein Chlor mehr nachzuweisen, wohl aber geringe Mengen von Silber; es erklart sich dies daraus, dafs die starke Salpetersaure beim Kochen geringe Mengen von Chlor aus dem Chlorsilber austreibt, welches sich bei dieser Temperatur verflüchtigt. Infolgedessen bleibt der gefundene Wert etwas hinter dem berechneten zurück.

Das äufsere der Terbindung, sowie der Gehalt an Chlorsilber erinnerten lebhaft an das Thiosinaminchlorsilber, welches Falke beschreibt. Zum weiteren Vergleiche mit Thiosinaminchlorsilber stellte ich mir eine Quantität dieser Verbindung durch Kochen von 
Thiosinaminlösung mit Chlorsilber dar. Dabei bemerkte ich, dafs Thiosinamin in der Kälte fist gar nicht auf das Chlorsilber einwirkte; erst wenn eine gewisse Temperatur erreicht war, füllte sich auf einmal die gesamte Flüssigkeit mit Hockigen Krystallmassen, wobei sie alkalische Reaktion annahm. Zur Lösung derselben ist es erforderlich, dals Thiosinamin im Ueberschuls vorhanden ist. Es scheiden sich alsdann im heirsen Filtrat allmählich so reichliche Mengen dieser federigen Krystallmassen ab, dals die gesamte Flüssigkeit zu einem Brei gesteht. Das durch Absangen und Trocknen über Schwefelsäure erzielte Präparat glich in seinem Aeufseren vollstandig dem obigen, durch Reduktion des Jodids und darauf folgendes Behandeln mit Chlorsilber erhaltenen Körper. Der Schmelzpunkt lag allerdings um $2-3^{0}$ höher, doch dürfte der niedrigere Schmelzpunkt des obigen Präparates durch geringe Verunreinigungen bedingt sein.

Durch Behandeln mit Annmoniak wurden beide Präparate bereits in der Kălte geschwärzt. Es schien demnach kaum zweifellaft zu sein, dafs beide Körper mit einander identisch seien. Zum weiteren Nachweis der Identität zerlegte ich beide Verbindungen mit Schwefelwasserstoff; im Filtrat war bei beiden noch Silber nachzuweisen, welches orst auf Zusatz von Schwefelammonium vollständig ausfiel. Zur Trockne eingedampft und mit Aether extrahiert, schieden beide weilse, durchsichtige Krystalle aus, die nach mehrmaligem Umkrystallisieren bei ca. $72^{\circ}$ schmolzen, also aus Thiosinamin bestanden. Verschieden war nur die Bildungsweise der beiden Verbindungen; denn während, wie gesagt, Thiosinamin sich erst in der Wärme mit Chlorsilber verbindet, wirkt das Reduktionsprodukt bereits in der Kälte auf das Chlorsilber ein, wie man aus der zahen Beschaffenheit orsehen kann, welche letzteres im Moment des Eintragens annimmt. Es scheint mir wabrscheinlich zu sein, dafs dieses verschiedene Verhalten dadurch bedingt ist, dafs sich in dem Reduktionsprodukt bereits eine Zinksalzverbindung des Thiosinamins befindet, welche sich mit Chlorsilber natürlich leicht umsetzen kann.

Weiterhin versuchte ich auch die Kupferchloridverbindung aus dem Reduktionsprodukt darzustellen. $Z$ u diesem $Z$ weck benutzte ich das mit Zink und Essigsäure reduzierte Thiosinamin b r o m id. Das klare Filtrat hierron gab sofort, nach dem Verdünnen mit 
Wasser, auf Zusatz von Kupfercblorid einen voluminösen, weifsen Niederschlag, der nach dem Absaugen, Auswaschen und Trocknen sich als vollständig identisch erwies mit Thiosinaminkupferchlorür. Durch Schwefelwasserstoff und Schwefelammonium zerlegt, schied auch dieses Krystalle aus, die sich durch den Schmelzpunkt und das Verhalten gegen Quecksilberoxyd als Thiosinamin charakterisierten.

Bei der Reduktion mit Zink und Salzsăure in wässriger Lösung wachte ich dieselben Erfahrungen.

Die wässrige, reduzierte Flüssigkeit lieferte mit Chlorsilber direkt und in der Kälte reichliche Mengen weifser Krystallnadeln. Erst beim Erwärmen verschwanden dieselben, und es fand eine Abscheidung von Jodsilber statt; als dann das Filtrat hiervon von neuem mit Chlorsilber versetzt und gekocht wurde, resultierten reichliche Mengen von Thiosinaminchlorsilber. Ja, es gelang mir sogar, aus dem Reduktionsprodukt Thiosinamin selbst zu isolieren, indem ich die salzsaure Lösung direkt mit Schwefelammonium fällte, das Filtrat rom ausgeschiedenen Schwefelzink zur Trockne dampfte und dann mit Aether extrahierte. Die dabei erzielten Krystalle erwiesen sich nach mehrinaligem Umkrystallisieren durch den Schmelzpunkt als reines Thiosinamin. Es kann somit keinem $Z$ weifel unterliegen, dafs bei der Reduktion mit Zink und einer Sii ure in alkoholischer oder wässriger Lösung a us dem Thiosinaminbromidoder-jodid Thiosinamin zur ückgebildet wird; ob nebenbei noch andere Körper sich bilden, wage ich. nicht zu eutscheiden, möchte es aber aus folgendem Grunde glauben :

Das Platindoppelsalz des mit Natriumamalgam reduzierten Thiosinaminjodids war, wie ich oben gezeigt babe, kein einheitliches. Es bestand aus einem bellen, amorphen, sich zuerst abscheidenden Teil und einem orangegelben, fein krystallinischen, der sich in zweiter Linie abschied. Das Aeufsere dieses letzteren Salzes glich nun völlig dem von mir dargestellten Thiosinaminplatinchlorid, auch der Platingehalt stimmte annähernd mit dem des letzteren uberein; die sonstigen Verschiedenheiten halte ich für bedingt durch die Beimischung des hellgelben Doppelsalzes. Ich möchte daher annehmen, dafs auch bei der Reduktion mit Natriumamalgam neben anderen Körpern Thiosinamin zuräckgebildet wird. 
Dieses Terhalten gegen Reduktionsmittel muls als ein uberaus auffalliges bezeichnet werden; denn selbst angenommen, dals keine der oben für das Thiosinaminbromid angegebenen Konstitutionsformeln die richtige sei, so mufs doch unbedingt zugegeben werden, dafs durch Addition von zwei Atomen Brom die doppelte Biuduug der Allylgruppe aufgehoben sein mufs. Hs $_{3}$ ist daher nicht loicht, eine genügende Erklürung dafür zu finden, dafs naszierender Wasserstoff durch Eliminierung der Halogenatome die doppelte Bindung wieder herzustellen vermochte. Vielleicht verläuft die Einwirkung des Wasserstoffs im statu nascendi, bei Annahme der oben für das Thiosinaminbromid aufgestellten Formeln, in folgender Weise:

I.
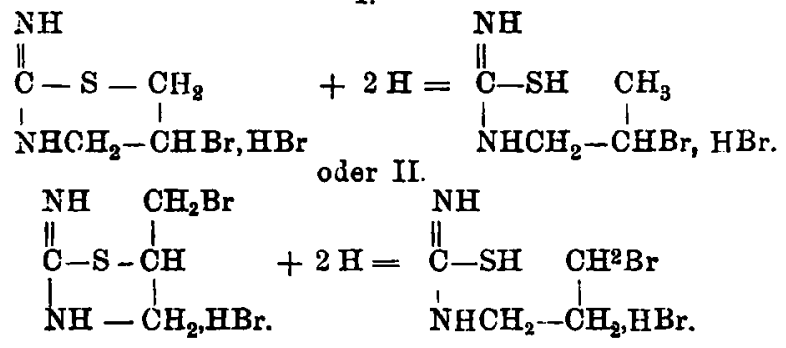

Die dabei entstehenden, um 2 Atome Wasserstoff reicheren Verbindungen sind aber unter den obwaltenden Bedingungen uicht existenzfähig, sondern spalten sich im Momente des Entstehens in Bromwasserstoft und Thiosinamin.

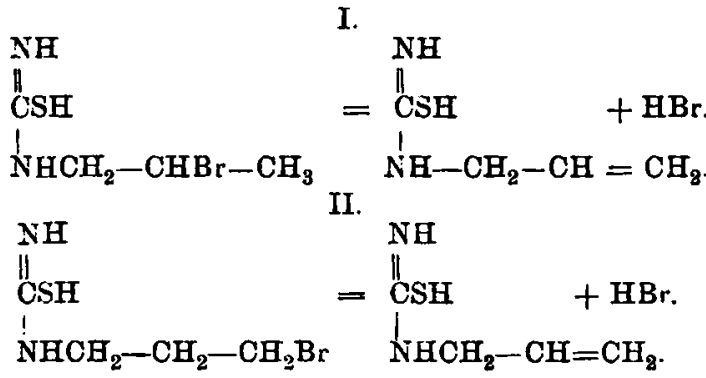

Die Wirkung des naszierenden Wasserstoffs würde demnn:h in einer Aufspaltung des Ringes bestehen; dafs eine derartige Wirkang wohl möglich sein wird, kann man daraus entnehmen, dafs eine ähnliche Aufspaltung auch durch Oxydation herbeigeführt wird, wie es Gabriel beim $\beta$ Metbylaethylen $\psi$ Thioharnstoff nachgewiesen 
hat, welcher bei der Oxydation in $\beta$ Methyltaurocarbaminsäure ubergeht. Fir die Entscheidung der Frage, welcher von beiden obigen Formeln der Forzug zu geben sei, ist dieses Verhalten insofern ron Wichtigkeit, als bei Annahme der F'ormel I nicht leicht einzusehen ist, warum der intermediür gebildete Körper, welcher mit dem durch Einwirkung rauchender Bromwasserstoffsäure auf Thiosinamin nach $\mathrm{Ga}$ bri el intermediär entstehenden Additionsprodukt identisch sein mülste, nicht, wie dieser, bei der Abspaltung von Bromwasserstoff in $\beta$-Methyläthylen- $\psi$-Thioharnstoff übergeht.

Nun liegt allerdings die Möglichkeit vor, dafs sich wohl intermediär letztere Verbindung gebildet, durch weitere Einwirkung von Wasserstoff aber eine Riickbildung zu Thiosinamin erfahren habe. War dem so, so mulste andererseits der Gabriel'sche Körper, in gleicher Weise behandelt, ebenfalls Thiosinamin liefern. Das war aber $n i c h$ t der Fall.

$\beta$-Methyläthylen- $\psi$-Thioharnstoff wurde in alkoholischer Lösung 5 Tage mit Zinkstaub und Essigsäure behandelt. Die Flüssigkeit nahm dadurch eine brännliche Farbe an. Ein Teil des Filtrats wurde mit wenig Chlorsilber gekocht; es trat keine sichtbare Veränderung ein. Auf Zusatz von Ammoniak entstand beim Kochen eine fast weifse, voluminöse Verbindung. Thiosinamin oder reduziertes Thiosinaminbromid bildete unter gleichen Umständen Thiosinaminchlorsilber und beim Erhitzen mit Ammoniak Schwofelsilber.

Ein anderer Teil wurde mit Kupferchlorid versetzt: Es entstand dadurch zunächst keine Fällung; erst nach mehrstïndigem Stehen entstand ein voluminöser Niederschlag, der sich auf Zusatz von Ammoniak und durch Erhitzen damit fast vollständig auflöste. Es blieb nur eine geringe Menge eines braunen Körpers ungelöst.

Reduziertes Thiosinaminbromid gab mit Kupferchloridlösung sofort einen Niederschlag, der beim Kochen mit Ammoniak reichliche Mengen von Schwefelkupfer abschied.

Demnach war also der $\beta$-Methyläthylen- $p$-Thioharnstoff durch Reduktion nicht in Thiosinamin zuruckgeführt worden. Andrerseits mëchte ich hier das Verhalten gegen ammoniakalische Chlorsilberlösung hervorheben. Wie obon gesagt, ruft letztere in der Lösung des $\beta$-Methylathylen- $\psi$-Thioharnstoffs einen fast weifsen, voluminosen Niederschlag hervor. Einen Niederschlag von dem gleichen Aeulseren 
erhielt ich, wenn ich Thiosinaminbromochlorid oder -jodochlorid mit ammoniakalischer Chlorsilberlösung versetzte. Dieses Verhalten, wie die Unfähigkeit letzterer Verbindnngen, mit neutralem Chlorsilber zu reagieren, macht ihrerseits die nahe Verwandtschaft des $\beta$-Methylathylen- $\psi$-Thioharnstoffes mit dem Thiosinaminbromid oder -jodid im hohen Grade wahrscheinlich. Auf Grund dieser Thatsachen möchte ich daher der zweiten Formel den Vorzug geben und das Thiosinaminbromid resp. Jodid als Monobrom- resp. Monojodsubstitutionsprodukte des $\beta$-Methyläthylen- $\psi$-Thioharnstoffes ansprechen.

Gabriel hat seine Pseudothioharnstoffe auch durch Einwirkung von Rhodankalium auf bromwasserstoffsaures Bromalkylanin dargestellt. Es konnte daher eventuell gelingen, durch analoge*Behandlung von bromwasserstoffsaurem Dibromallylamin mit Rhodankalium einen $\beta$-Monobrommethyläthylen- $\psi$-Thioharnstoff $z u$ erhalten. Zu diesem Behufe erwärmte ich, nach Gabriel, eine konzentrierte, wässerige Lösung von $5 \mathrm{~g}$ Dibromallylaminbrombydrat mit $2 \mathrm{~g}$ Rhodankalium auf dem Wasserbade. Esresultierte auch hier zunächst einezähe Masse, in welcher sich das ausgeschiedene Bromkalium befand, wie es Gabriel bei der Darstellung des Aethylen- $\psi$-Thioharnstoffes erwahnt; aber während bei letzterem Körper das Einwirkungsprodukt bei weiterem Erwärmen zu einer krystallinischen, harten Kruste erstarrt, blieb bei der Einwirkung von Rhodankalium auf Dibromallylaminbromhydrat, selbst nach sechsstlundigem Erwärmen, die zähe Beschaffenheit bestehen.

Da aber eine Umwandlung bereits eingetreten war (eine Probe, in Wasser gelöst, gab mit Eisenchlorid nicht mehr die Rhodanreaktion), so extrahierte ich die zähe Masse mit heilsem, absoluten Alkohol. Beim Verdunsten verblieb ein gelblicher Sirup, der nicht krystallisierbar zu sein scheint; auch durch ein Kryställchen des Thiosinamindibromids, mit dem die entstandene Verbindung eventuell identisch sein konnte, wurde eine Krystallisation nicht angeregt.

Durch Ueberschichten der alkoholischen Lösung mit Aether wurden ebenfalls keine Krystalle hervorgerufen; es schied sich nur eine gelbliche, zähe, ölige Flüssigkeit ab.

Einen andern Teil fuhrte ich durch Chlorsilber in das Chlorhydrat tiber und verwandelto dasselbe in das Platinsalz. Dasselbo fiel amorph aus, löste sich beim Erwärmen auf, schied sich aber 
beim Erkalten wieder amorph ab, war bei $100^{\circ}$ ziemlich beständig und enthielt 28,27 Proz. Platin.

0,1185 hinterlielsen beim Glahen $0,0335 \mathrm{~g} \mathrm{Pt}$.

Das Thiosinaminbromochlorid erfordert 24,36Proz. Pt und ist fein krystallinisch.

Wiederholte Versuche, unter verănderten Bedingungen, haben stets das Gleiche ergeben. Das vom Bromkalium durch absoluten Alkohol befreite Reaktionsprodukt war in keinem Falle krystallisierbar; das Platinsalz war in seinen aulseren Eigenschaften stets gleich, war aber nie ganz einheitlich. Stets lagerte sich auf einer hellen amorphen Ausscheidung nach einiger Zeit ein orangegelber krystallinischer ab.

Die von einem solchen Präparat ausgeführten Analysen haben folgendes ergeben :

1. $0,145 \mathrm{~g}$ Subst. gaben $0,0406 \mathrm{~g} \mathrm{Pt}$.

2. 0,2159 $\mathrm{g}$ Subst. gaben $0,3303 \mathrm{~g} \mathrm{Ag} \mathrm{Cl}$ und $\mathrm{Ag} \mathrm{Br}$. $0,2784 \mathrm{~g}$ davon verloren in Chlorstrom $0,0226 \mathrm{~g}$. Gefund $\in \mathbf{n}$ :

I II

Pt $28,00 \quad-$

$\mathrm{Cl} \quad-\quad 25, \mathrm{it}$

$\mathrm{Br} \quad-\quad 21,62$
Quotientev

1

5,04

$1,8.8$

Diese Daten beweisen, dafs kein einheitliches Produkt vorliegt. Aulserdem konnte ich stets geringe Mengen von Kalium im Glührückstande nachweisen, so dafs die Analysen zu unzuverlässig sind, um einen sichern Schluss auf die Zusammensetzung der entstandenen Verbindung ziehen zu können. Zweifellos aber ist sie nicht identisch mit dem Thiosinaminbromochlorid-Platinchlorid.

Da sie somit keinen Beitrag zur Entscheidung der Frage zu liefern vermag, habe ich die weitere Untersuchung aufgegeben.

Oxydationsversuche.

1. Oxydation des Thiosinaminbromids mit Bromwasser.

Gabriel erhielt bei der Oxydation seines $\beta$-Methyl-athylen$\psi$-Thioharnstoffs mit Bromwasser zunächst einen voluminösen Niederschlag, der sich beim Erwärmen wieder auflöste. Beim Verdunsten 
verblieb ein Sirup, der, in wenig warmem Wasser gelöst, beim Erkalten weifse Krystalle von $\beta$-Methyltaurocarbaminsäure ausschied.

Als ich das Thiosinaminbromid in derselben Weise behandelte, erhielt ich ebenfalls einen voluminösen Niederschlag, der sich aber beim Erwärmen nicht wieder völlig auflöste; vielmehr ballte sich der grïfste Teil zu schwach gelblich gefärbten, leicht zerreiblichen Massen zusammen. Letztere filtrierte ich von dem Gelösten ab, wusch mit Wasser nach und trocknete sie erst bei gewöhnlicher Temperatur, dann bei $100^{\circ}$. Dabei bemerkte ich, dafs der Körper stark ätzende Eigenschaften besals: Das Papier, zwischen dem es getrocknet wurde, nahm eine brüchige Beschaffenheit an; feuchtes, laues Lackmuspapier wurde intensiv gerötet.

Der Körper besals also die Eigenschatten einer Säure, hatte aber, wie untenstehende Analysen lehren, keine konstante Zusammensetzung. Einen sichern Schluls auf die Natur des Körpers vermag ich demnach nicht zu ziehen, nur scheint aus den gewonnenen Daten soviel mit Sicherheit hervorzugehen, dafs Bromwasser auf das Thiosinaminbromid nicht blos oxydierend einwirkt, sondern auch bromsubstituierend und tiefer zersetzend; denn je nach der Einwirkungsdauer des Bromwassers erbielt ich Körper, die, bei sonst äufserst ähnlichen physikalischen Eigenschaften, in Bromgehalt zwischen 35,2 und 45,66 Proz., im Stickstoff zwischen 8,13 und 14,07 Proz., im Schwefelgehalt zwischen 9,34 und 10,95 Proz. schwankten.

Um mich zu überzeugen, dafs ich das Thiosinaminbromid in analoger Weise behandelte, wie Gabriel den $\beta$-Methyl-äthylen$\psi$-Thioharnstoff, oxydierte ich einige Gramm letzteren Körpers genau in derselben Weise, wie mein Thiosinaminbromid. Ich fand dabei nur die Angaben G a b r i el's bestätigt, indem ich mit leichter Mühe zum Oxydationsprodukt, $\operatorname{der} \beta$ - Methyltaurocarbaminsäure gelangte. Letztere sintert bei $196^{\circ}$ und schmilzt unter Gasentwickelung bei 198-2050. Der obige amorphe Körper schmolz überhaupt nicht, sondern verkohlte nur beim Erhitzen auf dem Platinblech.

Von dem bei $100^{\circ}$ getrockneten Korper habe ich folgende Analysen ausgeführt:

1. Mit Bromwasser jn starkem Ueberschufs auf ein kleines Volumen eingedampft. 
a) 0,2533 g Substanz gaben 0,265 $\mathrm{g} \mathrm{Ag} \mathrm{Br}$ und $0,1723 \mathrm{~g} \mathrm{Ba} \mathrm{SO}_{4}$.

b) $0,3060 \mathrm{~g}$ Substanz lieferten $0,2433 \mathrm{~g} \mathrm{CO}_{2}$ und $0,0808 \mathrm{~g} \mathrm{H}_{2} \mathrm{O}$.

c) $0,1923 \mathrm{~g}$ gaben (nach Dumas) $23,5 \mathrm{ccm}$ Stickstoff bei $748 \mathrm{~mm}$ Druck und $150 \mathrm{C}$.

2. Wie sub 1 dargestelit.

$0,1952 \mathrm{~g}$ gaben $0,1987 \mathrm{~g} \mathrm{Ag} \mathrm{Br}$ und $0,1400 \mathrm{~g} \mathrm{Ba} \mathrm{SO}$.

3 . Wie obiges dargestellt.

0,215 g, mit Natriumkarbonat geglüht, ergaben nach dem Ansäuern mit Salpetersäure auf titrimetrischem Wege 45,66 Proz. Brom.

4. Mutterlaugen von der Darstellungsweise des Thiosinaminbromids mit überschüssigem Brom oxydiert und auf die Hälfte eingedampft.

a) $0,3678 \mathrm{~g}$ Substanz lieferten $0,2592 \mathrm{~g} \mathrm{CO}_{2}$ and $0,0883 \mathrm{~g} \mathrm{H}_{2} \mathrm{O}$.

b) $0,3781 \mathrm{~g}$ gaben (nach Dumas) $26,7 \mathrm{ccm} \mathrm{N}$ bei $14^{\circ} \mathrm{C}$ und $74 \mathrm{~m} / \mathrm{m}$ Druck.

5. Filtrat vom ausgeschiedenen Oxydationsprodukt im Exsiccator verdunstet und mit Wasser verdünnt. Diø dabei sich ausscheidenden anorphen Massen wurden bei $100^{\circ}$ getrocknet und analysiert.

a) 0,2002 g Substanz gaben $0,1650 \mathrm{~g} \mathrm{CO}_{2}$ und $0,0520 \mathrm{~g} \mathrm{H}_{2} \mathrm{O}$.

b) $0,1516 \mathrm{~g}$ gaben (nach Dumas) $15,1 \mathrm{ccm} \mathrm{N}$ bei $190 \mathrm{C}$. und $750 \mathrm{~m} / \mathrm{m}$ Druck.

c) $0,0781 \mathrm{~g}$ gaben nach Carius $0,0790 \mathrm{~g} \mathrm{Ag} \mathrm{Br}$.

6. Die wässrige Lösung des Bromids wurde mit überschüssigero Bromwasser versetzt und nur bis zur Entfärbung erwärmt. Das Ausscheidungsprodukt wurde dann sofort abfiltriert und nach dem Trocknen analysiert.

a) $0,2820 \mathrm{~g}$ Substanz lieferten $0,2265 \mathrm{~g} \mathrm{CO}_{2}$ und $0,0766 \mathrm{~g} \mathrm{H}_{2} \mathrm{O}$.

b) $0,3327 \mathrm{~g}$ nach Kjeldahl behandelt. Das daraus gewonnene Ammoniak saturierte $29,00 \mathrm{ccm} 1 / 10 \mathrm{~N}$. Salzsäure.

c) $0,2252 \mathrm{~g}$ gaben nach Carius $0,1863 \mathrm{~g}$ Ag Br und $0,1869 \mathrm{~g}$ $\mathrm{Ba} \mathrm{SO}_{4}$.

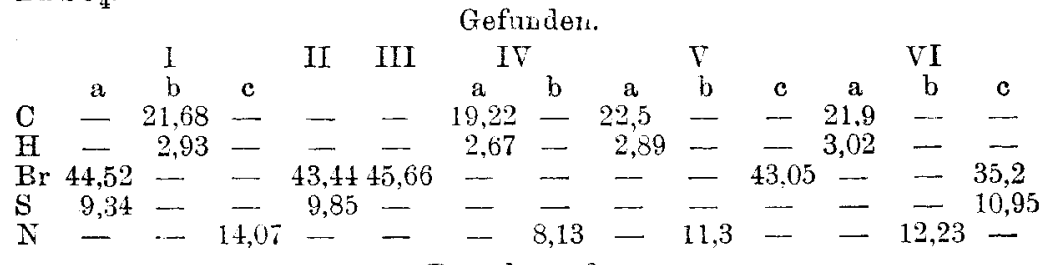

Berechnet für

Monobrom- Dibrom-

Methyltaurocarbaminsäure.

$\begin{array}{lrr}\mathrm{C} & 18,35 & 14,12 \\ \mathrm{H} & 3,45 & 2,35 \\ \mathrm{Br} & 30,65 & 47,06 \\ \mathrm{~S} & 12,26 & 9.41 \\ \mathrm{~N} & 10,73 & 8.24\end{array}$


Das Filtrat von dem beim Eindampfen unlöslichen Körper, welches auf Zusatz von Chlorbaryum einen reichlichen Niederschlag von Baryumsulfat gab, wurde auf dem Wasserbade auf ein kleines Volumen gebracht und dann über Aetzkalk im Vacuum verdunstet. Es verblieb ein bräunlicher Sirup, der durch Reiben nicht zum Krystallisieren gebracht werden konnte. An der Luft zog derselbe Feuchtigkeit an und wurde dünnflïssig, wahrscheinlich infolge des Gehaltes an freier Schwefelsäure. Beim Verdürnen mit Wasser schieden sich amorphe Massen aus, die sich beim Eindampfen wieder Iösten. Durch wiederholtes Auflösen in viel Wasser, Filtrieren und Verdunsten bis zum dicken Sirup wurden die amorphen Massen vollständig entfernt. Der zurückbleibende Sirup war als solcher nicht analysierbar. Ich versuchte ihn daher durch Ueberführung in das Silbersalz in analysierbare Form zu bringen. Zu diesem Behufe wurde die verdünnte Lösung mit Silbernitrat im Ueberschulis versetzt, das dabei sich ausscheidende Bromsilber abfiltriert, und das Filtrat vorsichtig mit Ammoniak neutralisiert. Dabei schied sich ein voluminöser, amorpher Niederschlag aus, der nach dem Absaugen, über Schwefelfäure getrocknet wurde. Dabei schrumpfte er zu einer hornartigen, schwärzlich gefärbten Masse zusammen, die von dem anhaftenden Filtrierpapier nur unvollkommen getrennt werden konnte.

$0,3162 \mathrm{~g}$ hinterliefsen beim Glühen im Wasserstoffstrome $0,0713 \mathrm{~g}$ metallisches Silber.

$$
\begin{array}{cc}
\text { Gefunden: } & \text { Berechnet für } \mathrm{C}_{4} \mathrm{H}_{8} \mathrm{BrN}_{2} \mathrm{SO}_{4} \cdot \mathrm{Ag} \\
\mathrm{Ag} 22,55 & 29,35 .
\end{array}
$$

Aus vorstehenden Beobachtungen geht hervor, dafs sich das Thiosinaminbromid anders, wie der $\beta$ Methylatbylen- $\psi$.Thioharnstoff bei der Oxydation mit Bromwasser verhält. Vor allem bezeichnend ist, meiner Ausicht nach, dals Brom hierbei zwar oxydierend wirkt, zugleich aber auch substituierend, während der $\beta$ Methylaethylen$\psi$-Thioharnstoff nur oxydiert wird.

\section{Oxydation des Thiosinaminbromids mit chlorsaurem Kali und Salzsänre.}

Eine äbnliche Wabrnehmung hat Prager bei der Oxydation der $\psi$ Thioharnstoffe, welche einen aromatischen Rest enthalten, bemerkt; auch er erhielt dabei stets ein bromhaltiges Produkt. Hingegen verlief die Oxydation verbaltnismälsig glatt bei Anwendung 
von chlorsaurem Kali und Salzsäure, zumal wenn nicht zu grolise Portionen auf einmal dargestellt wurden.

Ich verfubr deshalb genau nach seiner Vortchrift. Je $5 \mathrm{~g}$ Thiosinaminbromochlorid wurden in $25 \mathrm{ccm}$ Wasser gelöst, mit $25 \mathrm{ccm}$ rauchender Salzgäure versetzt und allmälig mit der berechneten Menge zerriebenen Kaliumchlorats $(2,7 \mathrm{~g})$ behandelt.

Beim jedesmaligen Eintragen entstand eine braungelbe Färbung, die aber allmalig in blafugelb uberging. Nach vollendeter Einwirkung wurde das Reaktiousgemisch bis nahezu zur Trockne bei måsiger Wärme eingedampft und vom ausgeschiedenen Chlorkalium durch Extraktion mit Alkohol nach Möglichkeit befreit. Beim Verdunsten der alkoholischen Lösung verblieb ein schwach gelb gefärbter Sirup, der beim Reiben mit einem Glasstab zu einer festen Krystallmasse erstarrte. Nach dem Absaugen auf Thontellern wurde das weifse Pulver aus Wasser umkrystallisiert, wobei es in derben Krystallen erhalten wurde.

Dieselben reagierten stark sauer und schmolzen unter Aufbransen bei ca. 210 . Die Ausbeute betrug etwa 40 Proz.

Die davon ausgetührten Analysen haben folgendes ergeben :

1. 0,5480 gaben $0,3370 \mathrm{CO}_{2}$ und $0,1398 \mathrm{H}_{2} \mathrm{O}$.

2. 0,3812 lieferten $0.3130 \mathrm{~g} \mathrm{Ba} \mathrm{SO}$.

Die gleichzeitig ausgefuhrte Brombestimmung erwies sich uls unbrauchbar, da, wie sich später herausstellte, das Präparat aufser Brom auch Chlor enthielt.

3. $0,4494 \mathrm{~g}$ Substanz leferten $0,5016 \mathrm{~g} \mathrm{Ag} \mathrm{Cl}+\mathrm{Ag} \mathrm{Br}, 0.4443 \mathrm{~g}$ davon verloren in Chlorstrome 0,0604 $\mathrm{g}$.

Gefunden :

$\begin{array}{lccc} & \text { I } & \text { II } & \text { III } \\ \text { C. } & 16,78 & - & - \\ \text { H. } & 2,83 & - & - \\ \text { S. } & - & 11,28 & - \\ \text { Br. } & - & - & 27,26 \\ \text { Cl. } & - & - & 11,74\end{array}$

Berechnet für: $\mathrm{C}_{4} \mathrm{H}_{8} \mathrm{Br} \mathrm{Cl} \mathrm{N} \mathrm{SO}_{4}$

] 6,24

2,71

10,83

27,07

12,00

Demnach ist die Verbindung als eine $\beta$-Chlorbrom-Methyltaurocarbaminsäure aufzufassen. Neben der Oxydation hat aber auch hier eine Substitution durch ein Atom Chlor stattgefunden War uun diese Säure von gleicher Struktur, wie die $\beta$-Metbyltaurocarbaminsäure Gabriels, so mulste es eventuell gelingen, zu 
letzterer selbst durch Einwirkung von naszierendem Wasserstoff zu gelangen.

Demgemäfs wurden $10 \mathrm{~g}$ Säure in Wasser gelöst und mit so viel granuliertem Zink versetzt, dafs von letzterem das $2 \frac{1}{2}$ fache der theoretiscben Menge vorbanden war. Unter äulserst lebhafter Wasserstoffentwickelung begann sich das Zink zu lösen, und es wurde erst dann verdünnte Schwefelsăure zugesetzt, als die Einwirkung langsamer zu werden begann. Nachdem alles Zink gelöst war, wurde dio etwas eingedampfte Flüssigkeit mit viel Alkohol von 96 Proz. versetzt und nach eintägigem Stehen vom ausgeschiedenen Zinksulfat durch Filtration getrennt. Um gelöstes Chlor- und Bromzink zu entfernen wurde die wiederum eingedampfte Flüssigkeit mit Sillerkarbonat im Ueberschufs kurze Zeit gelinde erwärmt; im Filtrat wurde das gelöste Silber und geringe Mengen noch vorhandenen Zinks durch Schwefelammonium und die dadurch gebildeten Ammoniumsalze durch Platinchlorid in alkoholisch-ätherischer Lösung entfernt. Das überschüssige Platinchlorid wurde durch Schwefelwasserstoff gefällt, und das Filtrat zur Krystallisation eingedampft. Nach einiger Zeit schieden sich fast weifse Krystalle aus, die in ihrer Form, Löslichkeitsverhältnissen und Schmelzpnnkt durchaus mit der $\beta$-Methyltaurokarbaminsäure übereinstimmten.

Die Analyse bestätigte die Identität.

1. $0.2358 \mathrm{~g}$ gaben nach Carius $0,2990 \mathrm{~g} \mathrm{Ba} \mathrm{SO}$.

2. $0,2774 \mathrm{~g}$ gabon $0,2662 \mathrm{~g} \mathrm{CO}_{2}$ und $0,1375 \mathrm{H}_{2} \mathrm{O}$.

Gefunden :

I II

$\begin{array}{llr}\text { S } & 17,51 & - \\ \text { C } & - & 26,17 \\ \text { H } & - & 5,51\end{array}$
Borechnet für

$\mathrm{C}_{4} \mathrm{H}_{10} \mathrm{~N}_{8} \mathrm{SO}_{4}$

$1 \bar{i}, 58$,

26,37 ,

5,49 .

Zum Schlufs versuchte ich die so erhaltene $\beta$-Methyltaurokarbaminsäure nach dem Vorgange Gabriel's in das $\beta$-Methyltaurin überzuführen.

$\mathrm{Zu}$ diesem Bebufe wurden 1,5 g Säure mit $6 \mathrm{~g}$ krystallisiertem Baryt und $6 \mathrm{ccm}$ Wasser im zugeschmolzenen Rohr 4 Stunden auf 140-1500 erhitzt. Beim Oeffnen des Rohres zeigte sich fast kein Druck. Der Inhalt, welcher stark nach Ammoniak roch, wurde zum Kochen erhitzt, mit Kohlensüure gesättigt und nach dem Filtieren mit einigen Tropfen Schwefelsäure versetzt. Befremdender Weise 
jedoch konnte die eingeengte Flüssigkeit nicht direkt zur Krystallisation gebracht werden; vielmebr verblieb ein sauer reagierender Sirup, in dem allerdings auf Zusatz eines kleinen Kryställchens ron $\beta$-Methyltaurin eine reichliche Krystallisation angeregt wurde; beim Versuche aber, dieselben durch Abpressen zwischen Thonplatten zu isolieren, lösten sich dieselben in der äufserst hygroskopischen Verunreinigung wieder auf.

Die Unlöslichkeit des $\beta$-Methyltaurins in Alkohol und Aether gaben mir jedoch ein Mittel in die Hand, dasselbe rein zu erhalten. Eine wässrige Lösung wurde successive mit dem 3 fachen Vol.Alkohol und einem Vol. Aether versetzt.

Nach kurzem Stehen schied sich das $\beta$-Methyltaurin in kleinen Tafeln und zu Drusen vereinigten Krystallen aus, die jetzt leicht aus Wasser krystallisierten.

Eine Bestinmung des Schwefelgehaltes bewies die Identität mit Gabriel's $\beta$-Methyltaurin.

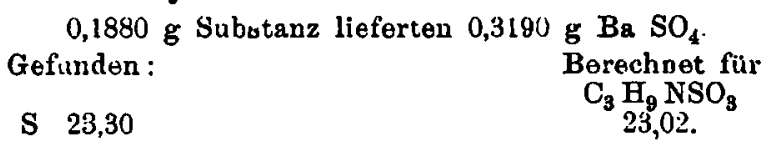

Somit ist der exakte Nachweis geliefert, dafs den $\mathrm{Ha}$ log $\mathrm{g}$ additionsprodukten des Thiosinamins dieselbe Struktur zukommt, wiedem $\beta$-Methylaethylen$\psi$-Thioharnstoff, dafs dieselben also nur als Monohalogensubstitutionsprodukte jenes Propylen $\psi$-ThioharastoffaGabrielsautzufasensind:<smiles>CC(C)SC=N</smiles>

Propylen $\psi$. Thiobarnstoff<smiles>N=C1NCC(Br)C1Br</smiles>

Tbiosinamindibromid

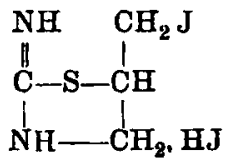

Thiosinamindijodid.

Einwirkung von Jodmethyl aut diefreie Base des Thiosinamindibromids.

Der $\beta$-Methyläthylen- $\psi$-Thioharustoff vereinigt sich mit Jodmethyl zu einer gut krystallisierten Verbindung, die bei $171-172^{\circ}$ schmilzt. F a lke hat die freie Base des Thiosinamindibromids mit Jodaethyl behandelt, ohne jedoch zu einem glatten Additionsprodukt gelangt 
zu sein. Da aber das Thiosinamindibromid, wie durch vorstehende Untersuchungen nachgewiesen ist, ein bromsubstituierter $\beta$-Methylaethylen $\psi$ Thioharnstoff ist, so ist dies abweichende Verhalten schwer zu begreifen und kann wobl nur in der Darstellungsweise seine Erklärung finden. Ich wiederholte daher den Tersuch mit Jodmethyl und verfuhr dabei folgendermafsen:

$5 \mathrm{~g}$ Thiosinaminbromid wurden in Methylalkohol gelöst und mit einer berechneten Menge methylalkoholischer Kalilauge und mit überschüssigem Jodmethyl versetzt. Nach mehrtägigem Stehen bei gewöhnlicher Temperatur war die alkalische Reaktion verschwunden, die Einwirkung also beendet. Die voin ausgeschiedenen Bromkalium abfiltrierte Flüssigkeit schied beim Verdunsten schön ausgebildete. glänzende Krystalle vom Schmelzpunkt $183-184^{\circ}$ aus. Eine Jod-und Brombestimmung bewies, dafs ein Jodmethylat des $\beta$. Brommethylaethylen- $\psi$-Thioharnstoffs vorlag.

$0,59+4 \mathrm{~g}$ Substanz gaben 0,7431 $\mathrm{g} \mathrm{AgBr}+\mathrm{AgJ}$. 0,6707 $\mathrm{g}$ davon verloren beim schwachen Glühen im Bromstrome $0,0 i 31 \mathrm{~g}$.

$\begin{array}{cc}\text { Gefunden: } & \text { Berechuet für } \mathrm{C}_{3} \mathrm{H}_{9} \mathrm{Br} \cdot \mathrm{N}_{2} \mathrm{~S}, \mathrm{HJ} \\ \mathrm{Br}: 2 t, 21 & 23, \pi \pm \\ \mathrm{J} 36,82 & 3 \overline{7}, \bar{\tau}\end{array}$

Mit Chlorsilber behandelt wurde Jod gegen Chlor ausgetauscht ; wio sich aber nachher herausstellte, war die Umwandlung keine quantitative, vielınehr blieb, selbst beim Eindampfen mit überschüssigem Chlorsilber bis zur Trockne, das Präparat jodhaltig. Dasselbe schmoli bei $1 \pi 1-1720$.

Hingegen - wurde der Verbindung durch Behandeln mit Silbernitrat in der Kälte alles Jod entzogen und gegen Chlor eingetauscht. Die nach Entfernen des uberschuissigen Silbernitrats mittelst Salzsäure dargestellten Gold- und Platindoppelsalze waren verhältnismärsig leicht löslich und krystallisierten in schön ausgebildeten Blätchen resp. Nadeln. Das Goldsalz schmolz bei 132-1330.

1. $0,2397 \mathrm{~g}$ Substanz gaben $0.0363 \mathrm{~g} \mathrm{Au}$

2. $0,3774 \mathrm{~g}$ " $, 0,1550 \mathrm{~g} \mathrm{CO}_{2}$ und $0,06+5 \mathrm{H}_{2} \mathrm{O}$.
Gefundən :
Berechnet für:

I. II.

$\Delta u 36,00$

$\mathrm{C}_{5} \mathrm{H}_{10} \mathrm{~N}_{2} \mathrm{SBrCl}$. $\mathrm{AuCl}_{3}$

$\mathrm{C} \quad-\quad 11,20$

35,83

H $\quad-\quad 1.89$

10,94

1,82 .

0,3306 g des Platinsalzes gaben 0,0768 g Pt. 
C. B o t tinger: Sulfometabrombenzoesäure. $\underset{\mathrm{Pt}}{\text { Gefunden : }} \quad$ Berechnet fur $\underset{23,50}{\left(\mathrm{C}_{3} \mathrm{H}_{10} \mathrm{~N}_{2} \mathrm{SBrCl}_{2} \mathrm{PtCl}_{4}\right.}$

Somit war eine Methylierung der Base erreicht.

Ihr kommt analog dem $\nu$.Methyl $\beta$-Methylaethylen $\psi$-Thioharnstoff die Strukturformel<smiles>CCC(C)SC(=N)N(C)C</smiles>

zu und ist mit der durch Einwirkung von Brom auf Methylthiosinamin entstandenen Base nicht identisch, wie auch die wesentlichen Differenzen in den Schmelzpunkten der verschiedenen Salzo lehren, sondern nur isomer.

Letzterer kommt die Formel

$$
\mathrm{CH}_{2} \mathrm{Br} \cdot \underset{\mid}{\mathrm{CH}}-\mathrm{S}
$$

zu.

\section{Ueber einige Abkömmlinge der Sulfometa- brombenzoesäure.}

Fon Dr. Carl B ottingor.

(Eingegangen den 19. XI. 1895.)

Vor Kurzem habe ich in der Chemiker-Zeitung ${ }^{1}$ ) die Methode heschrieben, nach welcher sich das Dichlorid der von mir schon im Jahre 1874 bereiteten Sulfoparabrombenzoësăure ${ }^{2}$ ) darstellen lä(st. Das Verfahren ist begründet auf die Wahrnehmung, dals beim Schütteln einer ätherischen oder petrolätherischen Lösung des Gemisches von Phosphoroxychlorid und Sulfochloridparabrombenzoylchlorid nit Wasser das Erstere zersetzt wird und dafs seine Umsetzungsprodukte mit Wasser von Letzterem gelöst werden, während das Chlorid der organischen Säure unter diesen Verhältnissen ziemlich geschützt und in den organischen Solventien gelöst bleibt.

Ich habe nunmehr auch das Dichlorid der Sulfometabrombenzoesăure, welches nach älteren Angaben ein Oel sein soll, nach

1) Chemiker-Zeitung 1895, 19 No. 75.

2) Berichte der d. chem. Gesellsch. 1874. 1781. 